\title{
Implications of $\mathcal{N}=5,6$ superconformal symmetry in three spacetime dimensions
}

\author{
Sergei M. Kuzenko and Igor B. Samsonov ${ }^{1}$ \\ School of Physics M013, The University of Western Australia, \\ 35 Stirling Highway, Crawley W.A. 6009, Australia \\ E-mail: sergei.kuzenko@uwa.edu.au, igor.samsonov@uwa.edu.au
}

ABSTRACT: For general $\mathcal{N}=5$ and $\mathcal{N}=6$ superconformal field theories in three dimensions, we compute the three-point correlation functions of the supercurrent multiplets. In each case, $\mathcal{N}=5$ and $\mathcal{N}=6$, the functional form of this correlator is uniquely fixed modulo an overall coefficient which is related, by superconformal Ward identities, to the parameter in the two-point function of the supercurrent. The structure of the correlation functions obtained is consistent with the property that every $\mathcal{N}=5$ superconformal field theory, considered as a special $\mathcal{N}=4$ theory, is invariant under the mirror map.

Keywords: Conformal Field Theory, Extended Supersymmetry, Superspaces

ArXIV EPRINT: 1605.08208

Dedicated to the memory of Professor Nikolay G. Pletnev

\footnotetext{
${ }^{1}$ On leave from Tomsk Polytechnic University, 634050 Tomsk, Russia.
} 


\section{Contents}

1 Introduction and summary 1

$2 \mathcal{N}=5$ superconformal field theories $\quad 4$

2.1 Correlators of $\mathcal{N}=5$ supercurrent 4

$2.2 \mathcal{N}=5 \rightarrow \mathcal{N}=4$ superspace reduction $\quad 5$

$\begin{array}{ll}2.3 \text { Superconformal theories in } \mathcal{N}=5 \text { superspace } & 7\end{array}$

$3 \mathcal{N}=6$ superconformal field theories $\quad 10$

$\begin{array}{ll}3.1 \text { Correlators of } \mathcal{N}=6 \text { supercurrent } & 11\end{array}$

$3.2 \mathcal{N}=6 \rightarrow \mathcal{N}=5$ superspace reduction $\quad 13$

3.3 Further $\mathcal{N}=5 \rightarrow \mathcal{N}=4$ superspace reduction $\quad 16$

$\begin{array}{llr}4 & \text { Discussion } & 18\end{array}$

$\begin{array}{ll}\text { A Two- and three-point building blocks } & 20\end{array}$

\section{Introduction and summary}

This is a continuation of our recent works $[1,2]$ in which the two- and three-point correlation functions of the supercurrents and flavour current multiplets in three-dimensional (3D) $\mathcal{N}$ extended superconformal field theories have been computed for the cases $1 \leq \mathcal{N} \leq 4$. The present paper extends the results of $[1,2]$ to the $3 \mathrm{D}$ field theories possessing $\mathcal{N}=5$ [3] and $\mathcal{N}=6[4,5]$ superconformal symmetry. The $\mathcal{N}=6$ superconformal field theories are often referred to as the $\mathrm{ABJ}(\mathrm{M})$ theories. ${ }^{1}$

Although the family of $\mathcal{N}$-extended superconformal field theories in three dimensions is very large for $\mathcal{N} \leq 4$, it becomes much smaller for $5 \leq \mathcal{N} \leq 8$. The latter families invariably consist of superconformal Chern-Simons theories interacting with supersymmetric matter in the bi-fundamental representation of the gauge group $G$ such that the amount of supersymmetry depends on the choice of $G$. The allowed gauge groups are as follows: $\mathrm{Sp}(2 M) \times \mathrm{O}(N)$ for $\mathcal{N}=5[3], \mathrm{U}(M) \times \mathrm{U}(N)$ or $\mathrm{Sp}(2 M) \times \mathrm{O}(2)$ for $\mathcal{N}=6[3,4]$, and only the gauge group $\mathrm{SU}(2) \times \mathrm{SU}(2)$ for $\mathcal{N}=8$ [8-10]. Clearly the range of $\mathcal{N}=5$ and $\mathcal{N}=6$ superconformal field theories are still pretty wide, and their properties are known to be quite fascinating.

In supersymmetric field theory in $d$ dimensions, the supercurrent [11] is a supermultiplet containing the energy-momentum tensor and the supersymmetry currents, along with some additional components such as the $R$-symmetry current. Thus the supercurrent contains fundamental information about the symmetries of every supersymmetric field theory.

\footnotetext{
${ }^{1}$ These theories possess the remarkably simple formulation [6] in $\mathcal{N}=3$ harmonic superspace [7].
} 
In the case of 3D extended superconformal field theories with $\mathcal{N}>4$, the supercurrent was introduced in [12, 13] (see also [1]). It is described by a primary real $\mathrm{SO}(\mathcal{N})$ four-form superfield $J^{I J K L}=J^{[I J K L]}$ of dimension $1, I=1, \ldots, \mathcal{N}$. The conformal supercurrent is subject to the conservation equation

$$
D_{\alpha}^{I} J^{J K L P}=D_{\alpha}^{[I} J^{J K L P]}-\frac{4}{\mathcal{N}-3} D_{\alpha}^{Q} J^{Q[J K L} \delta^{P] I}
$$

where $D_{\alpha}^{I}$ denotes the spinor covariant derivative. In the $\mathcal{N}=5$ case, it is convenient to replace the four-form $J^{I J K L}$ with its Hodge-dual one-form $J^{I}$ defined by

$$
J^{I}:=\frac{1}{4 !} \varepsilon^{I J K L P} J_{J K L P} .
$$

In terms of $J^{I}$, the conservation equation (1.1) turns into

$$
D_{\alpha}^{(I} J^{J)}-\frac{1}{5} \delta^{I J} D_{\alpha}^{Q} J^{Q}=0 .
$$

In the $\mathcal{N}=6$ case, it is useful to switch from $J^{I J K L}$ to its Hodge-dual two-form $J^{I J}$

$$
J^{I J}:=\frac{1}{4 !} \varepsilon^{I J K L P Q} J_{K L P Q} .
$$

In terms of $J^{I J}$, the conservation equation (1.1) turns into

$$
D_{\alpha}^{I} J^{J K}=D_{\alpha}^{[I} J^{J K]}-\frac{2}{5} D_{\alpha}^{Q} J^{Q[J} \delta^{K] I} .
$$

A remarkable property of $\mathcal{N}=6$ supersymmetry in three dimensions is that the supercurrent conservation equation (1.5) coincides with the Bianchi identity for an Abelian vector multiplet [13].

An important feature of the 3D extended superconformal theories with $\mathcal{N} \geq 5$ is the non-existence of conserved flavour current multiplets. This point has recently been discussed in [14], and here we follow almost verbatim the discussion in [14]. In supersymmetric field theory in $d$ dimensions, the conserved current multiplet is defined to be a supermultiplet containing a single conserved vector current $V^{a}$ (equivalently, a closed $(d-1)$-form), $\partial_{a} V^{a}=0$, along with some other scalar and spinor components. In three dimensions, one may think of a conserved current $V^{a}$ as the Hodge dual of the gauge-invariant field strength $F=\mathrm{d} A$ of a gauge one-form $A$. For this reason an $\mathcal{N}$-extended conserved current multiplet may be characterised by the same superfield type and the differential constraints as the field strength of an $\mathcal{N}$-extended Abelian vector multiplet $[7,15-19] .{ }^{2}$ Thus for $\mathcal{N}>2$, the conserved current multiplet should be defined to be a real antisymmetric superfield, $L^{I J}=-L^{J I}$, constrained by

$$
D_{\alpha}^{I} L^{J K}=D_{\alpha}^{[I} L^{J K]}-\frac{2}{\mathcal{N}-1} D_{\alpha}^{L} L^{L[J} \delta^{K] I} .
$$

For $\mathcal{N}>4$, it turns out that the off-shell multiplet constrained by (1.6) possesses more than one conserved current at the component level. Moreover, it also contains higher spin

\footnotetext{
${ }^{2}$ The conserved current multiplets with $\mathcal{N} \leq 4$ were reviewed in [1, 2].
} 
conserved currents for $\mathcal{N}>4[20,21]$. Indeed, for $\mathcal{N}=6$ the conservation equation (1.6) coincides with the supercurrent conservation equation (1.5). As a consequence, $L^{I J}$ contains a symmetric, traceless and conserved energy-momentum tensor $T^{a b}$

$$
T^{a b}=T^{b a}, \quad \eta_{a b} T^{a b}=0, \quad \partial_{b} T^{a b}=0,
$$

In the $\mathcal{N}=5$ case, $L^{I J}$ contains a conserved symmetric spinor current $S_{\alpha \beta \gamma}=S_{(\alpha \beta \gamma)}$ (supersymmetry current) defined by

$$
\left.S_{\alpha \beta \gamma} \propto \varepsilon_{I J K L M} D_{\alpha}^{I} D_{\beta}^{J} D_{\gamma}^{K} L^{L M}\right|_{\theta=0} .
$$

In this paper we find the most general expressions for the two- and three-point correlation functions of the $\mathcal{N}=5$ and $\mathcal{N}=6$ supercurrent multiplets, which are allowed by the superconformal symmetry and are compatible with the conservation equations (1.3) and (1.5), respectively. We show that the functional form of each of these correlators is determined by these requirements modulo a single overall coefficient to be denoted by $c_{\mathcal{N}}$ for the two-point functions and $d_{\mathcal{N}}$ for the three-point ones. The ratio of these coefficients turns out to be fixed by the superconformal Ward identities.

Every $\mathcal{N}=5$ or $\mathcal{N}=6$ superconformal field theory is a special $\mathcal{N}=4$ superconformal field theory. It is of interest to understand what additional restrictions on the structure of $\mathcal{N}=4$ correlation functions are implied by the $\mathcal{N}>4$ extended superconformal symmetry. For this we consider the reduction to $\mathcal{N}=4$ superspace of the obtained correlators of the $\mathcal{N}=5$ and $\mathcal{N}=6$ supercurrents and compare them with the results of the work [2]. It is worth recalling that in general $\mathcal{N}=4$ superconformal field theories the supercurrent threepoint function has two linearly independent functional structures with free coefficients $d_{\mathcal{N}}=4$ and $\tilde{d}_{\mathcal{N}=4}$. We demonstrate that one of these coefficients is equal to zero for all $\mathcal{N}$-extended superconformal field theories with $\mathcal{N}>4, \tilde{d}_{\mathcal{N}=4}=0$. In general, it was shown in [2] that $\tilde{d}_{\mathcal{N}=4}$ is non-zero in those $\mathcal{N}=4$ superconformal theories which are not invariant under the mirror map. As discussed in [2], $\tilde{d}_{\mathcal{N}=4}$ is proportional to the difference of the numbers of left and right hypermultiplets [2]. However, it will be demonstrated in section 2.3 that every $\mathcal{N}>4$ theory has an equal number of left and right hypermultiplets in the same representation of the gauge group. As a consequence, all $\mathcal{N}>4$ superconformal field theories are invariant under the mirror map.

As a by-product of the $\mathcal{N}=4$ superspace reduction of the $\mathcal{N}=5,6$ supercurrent correlation functions, we obtain new correlators in the $\mathcal{N}=4$ superspace which correspond to conserved currents of extended supersymmetry and $R$-symmetry. These results are presented in the next two sections, which are devoted to the $\mathcal{N}=5$ and $\mathcal{N}=6$ theories, respectively.

In the study of correlation functions we follow the superspace approach which was originally elaborated for $4 \mathrm{D} \mathcal{N}=1$ superconformal field theories in [22] and generalised to the $4 \mathrm{D} \mathcal{N}=2$ case in [23]. For 3D superconformal field theories this approach was originally developed in [24] and recently applied in [1,2] to study correlation functions of supercurrents and flavour current multiplets. In the present paper we use the superconformal formalism and definitions introduced in our works $[1,2]$, which are somewhat different from those adopted in [24]. The summary of our definitions is given in the appendix. 


\section{$2 \mathcal{N}=5$ superconformal field theories}

General $\mathcal{N}=5$ superconformal field theories are supersymmetric Chern-Simons-matter models with appropriately chosen interaction potentials $[3,5]$. In this section we compute the two- and three-point correlation functions of supercurrents in such theories and consider their reduction to $\mathcal{N}=4$ superspace.

\subsection{Correlators of $\mathcal{N}=5$ supercurrent}

As discussed in section 1 , the $\mathcal{N}=5$ supercurrent is a primary dimension- 1 superfield $J^{I}$ obeying the conservation equation (1.3). The two-point function of the supercurrent, which is compatible with this conservation law, reads

$$
\left\langle J^{I}\left(z_{1}\right) J^{J}\left(z_{2}\right)\right\rangle=c_{\mathcal{N}=5} \frac{u_{12}^{I J}}{\boldsymbol{x}_{12}{ }^{2}},
$$

where $c_{\mathcal{N}}=5$ is a free coefficient. Using the explicit form of the two-point structures $\boldsymbol{x}_{12}{ }^{2}$ and $u_{12}^{I J}$ given by (A.4) and (A.5), respectively, it is not hard to check that (2.1) obeys (1.3) at separate points, $z_{1} \neq z_{2}$.

We look for the three-point function $\left\langle J^{I}\left(z_{1}\right) J^{J}\left(z_{2}\right) J^{K}\left(z_{3}\right)\right\rangle$ in the form

$$
\left\langle J^{I}\left(z_{1}\right) J^{J}\left(z_{2}\right) J^{K}\left(z_{3}\right)\right\rangle=\frac{u_{13}^{I I^{\prime}} u_{23}^{J J^{\prime}}}{\boldsymbol{x}_{13}{ }^{2} \boldsymbol{x}_{23}{ }^{2}} H^{I^{\prime} J^{\prime} K}\left(\boldsymbol{X}_{3}, \Theta_{3}\right),
$$

where $H^{I J K}$ is a tensor depending on the three-point structures (A.8). Since the supercurrent has dimension 1 , this tensor should have the following scaling property

$$
H^{I J K}\left(\lambda^{2} \boldsymbol{X}, \lambda \Theta\right)=\lambda^{-2} H^{I J K}(\boldsymbol{X}, \Theta),
$$

for a real positive $\lambda$.

The supercurrent conservation law (1.3) implies that the tensor $H^{I J K}$ obeys the differential equation

$$
\mathcal{D}_{\alpha}^{(I} H^{J) K L}-\frac{1}{5} \delta^{I J} \mathcal{D}_{\alpha}^{M} H^{M K L}=0,
$$

where $\mathcal{D}_{\alpha}^{I}$ is the generalised covariant derivative (A.13).

The ansatz (2.2) can be applied to different orders of the operators $J^{I}\left(z_{1}\right), J^{J}\left(z_{2}\right)$ and $J^{K}\left(z_{3}\right)$. In particular, interchanging the order of the operators $J^{I}\left(z_{1}\right)$ and $J^{J}\left(z_{2}\right)$ in the correlator (2.2), one finds the following symmetry property of $H^{I J K}$

$$
H^{I J K}(\boldsymbol{X}, \Theta)=H^{J I K}\left(-\boldsymbol{X}^{\mathrm{T}},-\Theta\right)
$$

Similarly, swapping the operators $J^{I}\left(z_{1}\right)$ and $J^{K}\left(z_{3}\right)$ in $(2.2)$, one uncovers the constraint

$$
H^{I J K}\left(-\boldsymbol{X}_{1}^{\mathrm{T}},-\Theta_{1}\right)=\boldsymbol{x}_{13}{ }^{2} \boldsymbol{X}_{3}^{2} u_{13}^{J L} U_{3}^{L J^{\prime}} u_{13}^{I I^{\prime}} u_{13}^{K K^{\prime}} H^{K^{\prime} J^{\prime} I^{\prime}}\left(\boldsymbol{X}_{3}, \Theta_{3}\right) .
$$

This equation was derived with the help of identities (A.9). 
We find the general solution of the equations $(2.3)-(2.5)$ in the form:

$H^{I J K}=4 d_{\mathcal{N}=5} \varepsilon^{I J K L M} \frac{A^{L M}}{X^{3}}+d_{\mathcal{N}=5}\left(\delta^{I J} \varepsilon^{K L M N P}-\delta^{I K} \varepsilon^{J L M N P}-\delta^{J K} \varepsilon^{I L M N P}\right) \frac{A^{L M} A^{N P}}{X^{5}}$,

where $d_{\mathcal{N}=5}$ is a free coefficient and the matrix $A^{I J}$ is defined in (A.12). It is possible to show that the tensor (2.7) obeys also the equation (2.6) which ensures the invariance of the correlation function under the replacement $J^{I}\left(z_{1}\right) \longleftrightarrow J^{K}\left(z_{3}\right)$. In checking this, it is useful to express (2.7) in terms of the orthogonal matrix $U^{I J}$ given in (A.11)

$$
H^{I J K}=-\frac{1}{12} d_{\mathcal{N}=5}\left(H_{1}^{I J K}-8 H_{2}^{I J K}+H_{3}^{I J K}\right),
$$

where

$$
\begin{aligned}
& H_{1}^{I J K}=\left(\delta^{I K} \varepsilon^{J P Q R S}+\delta^{J K} \varepsilon^{I P Q R S}+U^{I J} \varepsilon^{K P Q R S}\right) \frac{U^{P Q} U^{R S}}{\boldsymbol{X}} \\
& H_{2}^{I J K}=\left(U^{I L} \varepsilon^{J L K P Q}-U^{L J} \varepsilon^{I L K P Q}-\varepsilon^{I J K P Q}\right) \frac{U^{P Q}}{\boldsymbol{X}} \\
& H_{3}^{I J K}=\varepsilon^{I J K L M} \frac{U^{L M} \Theta^{4}}{\boldsymbol{X}^{3}}
\end{aligned}
$$

Here we have used the following identities:

$$
\begin{aligned}
\boldsymbol{X}^{2} & =X^{2}+\frac{1}{4} \Theta^{4}, \\
\left(\varepsilon^{I L K M N} A^{L J}+\varepsilon^{J L K M N} A^{L I}\right) A^{M N}= & \frac{1}{4}\left(\delta^{I K} \varepsilon^{J M N P Q}+\delta^{J K} \varepsilon^{I M N P Q}\right. \\
& \left.-2 \delta^{I J} \varepsilon^{K M N P Q}\right) A^{M N} A^{P Q}, \\
A^{I J} \varepsilon^{K L M N P} A^{L M} A^{N P}= & -2 \varepsilon^{I J K P Q} A^{P Q} \Theta^{4} X^{2}+2\left(\Theta^{K} \Theta^{L}\right) \varepsilon^{I J L P Q} A^{P Q} \Theta^{2} X^{2} .
\end{aligned}
$$

The identity (2.10) follows from (A.10) while the other two are direct consequences of (A.12).

Using the identities (A.9) it is not hard to verify that each line in (2.9) obeys (2.6). This ensures the invariance of the obtained expression for the correlation function (2.2) under the interchange of operators $J^{I}\left(z_{1}\right)$ and $J^{K}\left(z_{3}\right)$.

\section{$2.2 \mathcal{N}=5 \rightarrow \mathcal{N}=4$ superspace reduction}

As discussed in section 1 , every $\mathcal{N}=5$ superconformal field theory is a special $\mathcal{N}=4$ one. The $\mathcal{N}=5$ supercurrent is equivalent to two $\mathcal{N}=4$ supermultiplets, one of which is the $\mathcal{N}=4$ supercurrent. As a result, the three-point function of the $\mathcal{N}=5$ supercurrent is equivalent to several three-point functions in $\mathcal{N}=4$ superspace. Here we elaborate on the $\mathcal{N}=5 \rightarrow \mathcal{N}=4$ superspace reduction of the $\mathcal{N}=5$ supercurrent and its correlation functions.

We split the Grassmann coordinates $\theta_{I}^{\alpha}$ of $\mathcal{N}=5$ Minkowski superspace $\mathbb{M}^{3 \mid 10}$ onto two subsets: (i) the coordinates $\theta_{\hat{I}}^{\alpha}$, with $\hat{I}=1, \ldots, 4$, corresponding to $\mathcal{N}=4$ Minkowski 
superspace $\mathbb{M}^{3 \mid 8}$; and (ii) two additional coordinates $\theta_{5}^{\alpha}$. The corresponding splitting of the spinor derivatives $D_{\alpha}^{I}$ is $D_{\alpha}^{\hat{I}}$ and $D_{\alpha}^{5}$. Given a superfield $V$ on $\mathbb{M}^{3 \mid 10}$, its bar-projection onto $\mathbb{M}^{3 \mid 8}$ is defined by $V|:=V|_{\theta_{5}=0}$. The $\mathcal{N}=5$ supercurrent $J^{I}$ reduces to the following $\mathcal{N}=4$ superfields:

$$
S^{\hat{I}}=J^{\hat{I}}\left|, \quad J=J^{5}\right|
$$

The $\mathcal{N}=5$ supercurrent conservation law (1.3) implies that $S^{\hat{I}}$ and $J$ obey the constraints

$$
\begin{aligned}
D_{\alpha}^{(\hat{I}} S^{\hat{J})}-\frac{1}{4} \delta^{\hat{I} \hat{J}} D_{\alpha}^{\hat{K}} S^{\hat{K}}=0, \\
D^{\hat{I} \alpha} D_{\alpha}^{\hat{J}} J-\frac{1}{4} \delta^{\hat{I} \hat{J}} D^{\hat{Q} \alpha} D_{\alpha}^{\hat{Q}} J=0 .
\end{aligned}
$$

Eq. (2.14b) tells us that $J$ is the $\mathcal{N}=4$ supercurrent [1,2]. The second multiplet, $S^{\hat{I}}$, contains among its components the current of the fifth supersymmetry and the currents of the remaining $\mathrm{SO}(5) / \mathrm{SO}(4) R$-symmetry.

Note that the two-point structure $u_{12}^{I J}$ is the integral part of two- and three-point functions (2.1) and (2.2). Its reduction to $\mathcal{N}=4$ superspace leads to

$$
u_{12(\mathcal{N}=5)}^{\hat{I} \hat{J}}\left|=u_{12(\mathcal{N}=4)}^{\hat{I} \hat{J}}, \quad u_{12(\mathcal{N}=5)}^{I 5}\right|=\delta^{I 5} .
$$

Here we have assigned the labels $(\mathcal{N}=4)$ and $(\mathcal{N}=5)$ to distinguish these structures in the corresponding superspace. Below we will omit these labels to simplify the notation.

Using the relations (2.15) we find the $\mathcal{N}=4$ superspace reduction of the two-point correlator (2.1)

$$
\begin{aligned}
\left\langle S^{\hat{I}}\left(z_{1}\right) S^{\hat{J}}\left(z_{2}\right)\right\rangle & =c_{\mathcal{N}=5} \frac{u_{12}^{\hat{I} \hat{J}}}{\boldsymbol{x}_{12}{ }^{2}}, \\
\left\langle J\left(z_{1}\right) J\left(z_{2}\right)\right\rangle & =\frac{c_{\mathcal{N}=5}}{\boldsymbol{x}_{12}{ }^{2}} .
\end{aligned}
$$

The latter correlation function coincides with the two-point correlator of the $\mathcal{N}=4$ supercurrent found in [2] provided we identify $c_{\mathcal{N}=4}=c_{\mathcal{N}=5}$.

The $\mathcal{N}=5$ supercurrent three-point correlator (2.2) reduces to the following four correlation functions of the $\mathcal{N}=4$ superfields $S^{\hat{I}}$ and $J$

$$
\left\langle S^{\hat{I}} S^{\hat{J}} S^{\hat{K}}\right\rangle, \quad\left\langle S^{\hat{I}} S^{\hat{J}} J\right\rangle, \quad\left\langle S^{\hat{I}} J J\right\rangle, \quad\langle J J J\rangle,
$$

which can be found from different projections of the tensor (2.7). In particular, since $H^{\hat{I} \hat{J} \hat{K}} \mid=0$ and $H^{\hat{I} 55} \mid=0$, two of the four correlation functions in (2.17) vanish

$$
\left\langle S^{\hat{I}} S^{\hat{J}} S^{\hat{K}}\right\rangle=0, \quad\left\langle S^{\hat{I}} J J\right\rangle=0 .
$$

The other two correlators are non-trivial. They are

$$
\begin{aligned}
\left\langle S^{\hat{I}}\left(z_{1}\right) S^{\hat{J}}\left(z_{2}\right) J\left(z_{3}\right)\right\rangle & =\frac{u_{13}^{\hat{I} \hat{I}^{\prime}} u_{23}^{\hat{J} \hat{J}^{\prime}}}{\boldsymbol{x}_{13}{ }^{2} \boldsymbol{x}_{23}{ }^{2}} H_{\mathcal{N}=4}^{\hat{I}^{\prime} \hat{J}^{\prime}}\left(\boldsymbol{X}_{3}, \Theta_{3}\right), \\
H_{\mathcal{N}=4}^{\hat{I} \hat{J}}=H^{\hat{I} \hat{J} 5} \mid & =4 d_{\mathcal{N}=5} \varepsilon^{\hat{I} \hat{J} \hat{K} \hat{L}} \frac{A^{\hat{K} \hat{L}}}{X^{3}}+d_{\mathcal{N}=5} \delta^{\hat{I} \hat{J}} \varepsilon^{\hat{K} \hat{L} \hat{M} \hat{N}} \frac{A^{\hat{K} \hat{L}} A^{\hat{M} \hat{N}}}{X^{5}},
\end{aligned}
$$


and

$$
\begin{aligned}
\left\langle J\left(z_{1}\right) J\left(z_{2}\right) J\left(z_{3}\right)\right\rangle & =\frac{H_{\mathcal{N}=4}\left(\boldsymbol{X}_{3}, \Theta_{3}\right)}{\boldsymbol{x}_{13}{ }^{2} \boldsymbol{x}_{23}{ }^{2}}, \\
H_{\mathcal{N}=4}=H^{555} \mid & =-d_{\mathcal{N}=5} \varepsilon^{\hat{I} \hat{J} \hat{K} \hat{L}} \frac{A^{\hat{I}} \hat{J} A^{\hat{K} \hat{L}}}{X^{5}} .
\end{aligned}
$$

We recall that the most general form of the function $H_{\mathcal{N}=4}$, which defines the correlation function of the $\mathcal{N}=4$ supercurrent, is [2]

$$
H_{\mathcal{N}=4}=\frac{\tilde{d}_{\mathcal{N}=4}}{X}-d_{\mathcal{N}=4} \varepsilon^{\hat{I} \hat{J} \hat{K} \hat{L}} \frac{A^{\hat{I} \hat{J}} A^{\hat{K} \hat{L}}}{X^{5}}
$$

where $d_{\mathcal{N}=4}$ and $\tilde{d}_{\mathcal{N}=4}$ are two independent coefficients. Comparing (2.21) with (2.20b) we make the following two conclusions: (i) only those $\mathcal{N}=4$ superconformal field theories for which

$$
\tilde{d}_{\mathcal{N}=4}=0
$$

may possess extended $\mathcal{N}>4$ supersymmetry; (ii) the coefficients $d_{\mathcal{N}=4}$ and $d_{\mathcal{N}=5}$ are equal,

$$
d_{\mathcal{N}=4}=d_{\mathcal{N}=5} .
$$

Recall that the coefficients $c_{\mathcal{N}=4}$ and $d_{\mathcal{N}=4}$ are related to each other by the superconformal Ward identity [2]. As a consequence of (2.23) the same identity holds for $c_{\mathcal{N}=5}$ and $d_{\mathcal{N}=5}$ :

$$
\frac{d_{\mathcal{N}=4}}{c_{\mathcal{N}=4}}=\frac{d_{\mathcal{N}=5}}{c_{\mathcal{N}=5}}=\frac{1}{16 \pi}
$$

In conclusion of this subsection, let us briefly comment on the condition (2.22) which is

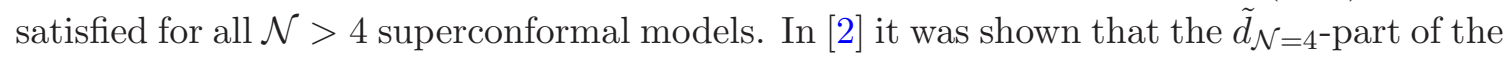
$\mathcal{N}=4$ supercurrent correlation function is non-trivial for those $\mathcal{N}=4$ models which have non-equal numbers of the left and right hypermultiplets (transforming in $(\mathbf{2}, \mathbf{0})$ and $(\mathbf{0 , 2})$ representations of the group $\mathrm{SU}(2)_{\mathrm{L}} \times \mathrm{SU}(2)_{\mathrm{R}}$ which is the double cover of the $\mathcal{N}=4 R$ symmetry group $\mathrm{SO}(4) \cong\left(\mathrm{SU}(2)_{\mathrm{L}} \times \mathrm{SU}(2)_{\mathrm{R}}\right) / \mathbb{Z}_{2}$. Thus, only those $\mathcal{N}=4$ superconformal field theories may possess extended $\mathcal{N} \geq 5$ supersymmetry which contain the same number of left and right hypermultiplets. In the next subsection we will confirm this statement by considering equations of motion of general $\mathcal{N}=5$ superconformal theories. We will demonstrate that every $\mathcal{N}=5$ superconformal field theory realised in $\mathcal{N}=4$ superspace has equal number of left and right hypermultiplets.

\subsection{Superconformal theories in $\mathcal{N}=5$ superspace}

The $R$-symmetry group of the $\mathcal{N}=5$ super-Poincare algebra is $\mathrm{SO}(5) \cong \mathrm{USp}(4) / \mathbb{Z}_{2}$, where the group USp $(4)$ consists of matrices $g=\left(g_{\mathrm{a}}{ }^{\mathrm{b}}\right) \in \mathrm{GL}(4, \mathbb{C})$ constrained by

$$
g^{\dagger} g=\mathbb{1}_{4}, \quad g^{\mathrm{T}} \Omega g=\Omega,
$$

for a given non-singular real symplectic metric $\Omega=\left(\Omega_{\mathrm{ab}}\right)=-\Omega^{\mathrm{T}}$. This tensor is used to raise and lower the USp(4) indices,

$$
X^{\mathrm{a}}=\Omega^{\mathrm{ab}} X_{\mathrm{b}}, \quad X_{\mathrm{a}}=\Omega_{\mathrm{ab}} X^{\mathrm{b}} .
$$

Here $\Omega^{-1}=\left(\Omega^{\mathrm{ab}}\right)$ is the inverse of $\Omega, \Omega_{\mathrm{ab}} \Omega^{\mathrm{bc}}=\delta_{\mathrm{a}}^{\mathrm{c}}$. 
We recall that the isomorphism $\mathrm{SO}(5) \cong \mathrm{USp}(4) / \mathbb{Z}_{2}$ can be established by making use of a set of gamma-matrices $\gamma_{I}=\left(\left(\gamma_{I}\right)_{\mathrm{a}}{ }^{\mathrm{b}}\right)$ for $\mathrm{SO}(5)$ with the properties

$$
\gamma_{I} \gamma_{J}+\gamma_{J} \gamma_{I}=2 \delta_{I J} \mathbb{1}_{4}, \quad \gamma_{I}^{\dagger}=\gamma_{I}, \quad \gamma_{I}^{\mathrm{T}} \Omega=\Omega \gamma_{I}, \quad I=1, \ldots, 5 .
$$

An explicit realisation for the matrices $\Omega$ and $\gamma_{I}$ is as follows:

$$
\Omega=\left(\begin{array}{cc}
\varepsilon & 0 \\
0 & \tilde{\varepsilon}
\end{array}\right), \quad \varepsilon=\left(\varepsilon_{i j}\right)=-\varepsilon^{\mathrm{T}}, \quad \tilde{\varepsilon}=\left(\varepsilon_{\tilde{i} \tilde{j}}\right)=-\tilde{\varepsilon}^{\mathrm{T}}, \quad \varepsilon_{12}=\varepsilon_{\tilde{1} \tilde{2}}=1
$$

and $\gamma_{I}=\left(\vec{\gamma}, \gamma_{4}, \gamma_{5}\right)$,

$$
\vec{\gamma}=\left(\begin{array}{cc}
0 & \mathrm{i} \vec{\sigma} \\
-\mathrm{i} \vec{\sigma} & 0
\end{array}\right), \quad \gamma_{4}=\left(\begin{array}{cc}
0 & \mathbb{1}_{2} \\
\mathbb{1}_{2} & 0
\end{array}\right), \quad \gamma_{5}=\left(\begin{array}{cc}
\mathbb{1}_{2} & 0 \\
0 & -\mathbb{1}_{2}
\end{array}\right),
$$

with $\vec{\sigma}$ the Pauli matrices. Here and below, we represent an USp(4) index as a pair of $\mathrm{SU}(2)$ ones, $X^{\mathrm{a}}=\left(X^{i}, X^{\tilde{i}}\right)$.

They allow one to establish an isomorphism between $\mathbb{R}^{5}$ and the following linear space $\mathcal{L}$ of $4 \times 4$ matrices $\hat{X}$ constrained by

$$
\hat{X}^{\dagger}=\hat{X}, \quad \hat{X}^{\mathrm{T}} \Omega=\Omega \hat{X}, \quad \operatorname{tr} \hat{X}=0 .
$$

The isomorphism between $\mathbb{R}^{5}$ and $\mathcal{L}$ is defined as follows: given a five-vector $\vec{X}=\left(X^{I}\right) \in$ $\mathbb{R}^{5}$, its image is $\hat{X}=X^{I} \gamma_{I} \in \mathcal{L}$. The group $\operatorname{USp}(4)$ naturally acts on $\mathcal{L}$ by nonsingular linear operators. Given a group element $g \in \mathrm{USp}(4)$, the corresponding transformation $\hat{g}$ on $\mathcal{L}$ is defined by $\hat{g}: \hat{X} \rightarrow g \hat{X} g^{-1}$. This induces a linear transformation $A(g)$ on $\mathbb{R}^{5}$ that preserves the inner product $\langle\vec{X} \mid \vec{Y}\rangle=\frac{1}{4} \operatorname{tr}(\hat{X} \hat{Y})$. It may be checked that the correspondence $g \rightarrow A(g)$ defines a homomorphism of $\operatorname{USp}(4)$ onto $\mathrm{SO}(5)$ with the kernel $\mathbb{Z}_{2}=\left\{ \pm \mathbb{1}_{4}\right\}$.

Using the symplectic metric $\Omega$ and its inverse $\Omega^{-1}$, let us introduce gamma-matrices with upper and lower indices

$$
\left(\gamma_{I}\right)^{\mathrm{ab}}=\Omega^{\mathrm{ac}}\left(\gamma_{I}\right)_{\mathrm{c}}^{\mathrm{b}}, \quad\left(\gamma_{I}\right)_{\mathrm{ab}}=\Omega_{\mathrm{bc}}\left(\gamma_{I}\right)_{\mathrm{a}}^{\mathrm{c}}=\overline{\left(\gamma_{I}\right)^{\mathrm{ab}}} .
$$

These matrices are antisymmetric and $\Omega$-traceless

$$
\left(\gamma_{I}\right)^{\mathrm{ab}}=-\left(\gamma_{I}\right)^{\mathrm{ba}}, \quad\left(\gamma_{I}\right)^{\mathrm{ab}} \Omega_{\mathrm{ab}}=0 .
$$

Thus, any $\mathrm{SO}(5)$ vector $X^{I}$ is equivalent to an antisymmetric $\Omega$-traceless second-rank spinor

$$
X^{\mathrm{ab}}:=\gamma_{I}^{\mathrm{ab}} X^{I}, \quad X^{I}=\frac{1}{4} \gamma_{\mathrm{ab}}^{I} X^{\mathrm{ab}} .
$$

Using the rule (2.32) we introduce the spinor covariant derivatives with $\mathrm{SO}(5)$ spinor indices, $D_{\alpha}^{I} \rightarrow D_{\alpha}^{\mathrm{ab}}=\gamma_{I}^{\mathrm{ab}} D_{\alpha}^{I}$. Their anti-commutation relations follow from (A.14),

$$
\left\{D_{\alpha}^{\mathrm{ab}}, D_{\beta}^{\mathrm{cd}}\right\}=2 \mathrm{i}\left(\Omega^{\mathrm{ab}} \Omega^{\mathrm{cd}}-2 \varepsilon^{\mathrm{abcd}}\right) \partial_{\alpha \beta} .
$$

Let us consider a gauge theory in the $\mathcal{N}=5$ superspace

$$
\partial_{\alpha \beta} \rightarrow \nabla_{\alpha \beta}=\partial_{\alpha \beta}+\mathrm{i} V_{\alpha \beta}, \quad D_{\alpha}^{\mathrm{ab}} \rightarrow \nabla_{\alpha}^{\mathrm{ab}}=D_{\alpha}^{\mathrm{ab}}+\mathrm{i} V_{\alpha}^{\mathrm{ab}},
$$


where $\left(V_{\alpha \beta}, V_{\alpha}^{\mathrm{ab}}\right)$ are gauge connections. To describe the vector multiplet, the gauge covariant derivatives are subject to a covariant constraint which implies

$$
\left\{\nabla_{\alpha}^{\mathrm{ab}}, \nabla_{\beta}^{\mathrm{cd}}\right\}=2 \mathrm{i}\left(\Omega^{\mathrm{ab}} \Omega^{\mathrm{cd}}-2 \varepsilon^{\mathrm{abcd}}\right) \nabla_{\alpha \beta}+\varepsilon_{\alpha \beta}\left(\Omega^{\mathrm{ac}} W^{\mathrm{bd}}-\Omega^{\mathrm{bc}} W^{\mathrm{ad}}-\Omega^{\mathrm{ad}} W^{\mathrm{bc}}+\Omega^{\mathrm{bd}} W^{\mathrm{ac}}\right) .
$$

Here $W^{\mathrm{ab}}=W^{\mathrm{ba}}$ is the field strength obeying the Bianchi identity

$$
\nabla_{\alpha}^{\mathrm{a}(\mathrm{b}} W^{\mathrm{cd})}+\frac{1}{3} \Omega^{\mathrm{a}(\mathrm{b}} \nabla_{\alpha}{ }^{\mathrm{c}}{ }_{\mathrm{e}} W^{\mathrm{d}) \mathrm{e}}=0 .
$$

In complete analogy with the $\mathcal{N}=6$ analysis in [18], we now consider a matter superfield $\Phi^{\mathrm{a}}$ in some representation of the gauge group. The equation of motion for the matter superfield is

$$
\nabla_{\alpha}^{\mathrm{a}(\mathrm{b}} \Phi^{\mathrm{c})}-\frac{1}{5} \Omega^{\mathrm{a}(\mathrm{b}} \nabla_{\alpha}^{\mathrm{c}) \mathrm{d}} \Phi_{\mathrm{d}}=0
$$

The consistency condition for the equation (2.37) is

$$
W^{(\mathrm{ab}} \Phi^{\mathrm{c})}=0 .
$$

To solve this constraint we should assume that $W^{\mathrm{ab}}$ is a composite of the matter superfields

$$
W^{\mathrm{ab}}=W_{A}^{\mathrm{ab}} T^{A}, \quad W_{A}^{\mathrm{ab}}=\mathrm{i} \kappa g_{A B} \bar{\Phi}^{(\mathrm{a}} T^{B} \Phi^{\mathrm{b})},
$$

where $\kappa$ is some coefficient, $T^{A}$ are generators of the representation and $g_{A B}$ is an invariant quadratic form on the Lie algebra of the gauge group. Note also that the Hermitian conjugate for $\Phi^{\mathrm{a}}$ is $\bar{\Phi}_{\mathrm{a}}=\left(\Phi^{\mathrm{a}}\right)^{\dagger}$, where we assume that $\Phi^{\mathrm{a}}=\left(\Phi_{p}^{\mathrm{a}}\right)$ is a column vector in some representation of the gauge group and the letters $p, q, r, s$ from the middle of Latin alphabet denote gauge indices.

Substituting (2.39) into the consistency condition (2.38) we find

$$
g_{A B} \bar{\Phi}^{p(\mathrm{a}} \Phi_{q}^{\mathrm{b}} \Phi_{s}^{\mathrm{c})}\left(T^{B}\right)_{p}^{q}\left(T^{A}\right)_{r}{ }^{s}=0,
$$

or

$$
g_{A B}\left(T^{A}\right)_{p}{ }^{(q}\left(T^{B}\right)_{r}{ }^{s)}=0 .
$$

The latter equation imposes strong constraints on the possible gauge group and its representations. These constraints were analysed in the works $[3,5]$ where the admissible gauge groups were classified.

Let us consider the $\mathcal{N}=5$ supercurrent $J^{I}$ in the USp(4) spinor notation $J^{\mathrm{ab}}=\gamma_{I}^{\mathrm{ab}} J^{I}$. The conservation law (1.3) turns into

$$
D_{\alpha}^{\mathrm{ab}} J^{\mathrm{cd}}+D_{\alpha}^{\mathrm{cd}} J^{\mathrm{ab}}+\frac{1}{10}\left(\Omega^{\mathrm{ab}} \Omega^{\mathrm{cd}}-2 \varepsilon^{\mathrm{abcd}}\right) D_{\alpha}^{\mathrm{ef}} J_{\mathrm{ef}}=0 .
$$

For the $\mathcal{N}=5$ superconformal theories described by the equations (2.37) and (2.39), we find the following expression for the supercurrent in terms of the matter superfields

$$
J^{\mathrm{ab}}=\bar{\Phi}^{[\mathrm{a}} \Phi^{\mathrm{b}]}+\frac{1}{4} \Omega^{\mathrm{ab}} \bar{\Phi}^{\mathrm{c}} \Phi_{\mathrm{c}} .
$$


It is possible to check that this expression obeys (2.42) due to the equations of motion (2.37) and (2.39).

Now we consider the $\mathcal{N}=4$ superfield reduction of $\mathcal{N}=5$ superconformal models described above. This reduction amounts to setting $\theta_{\alpha}^{5}=0$ in the superfields $\Phi^{\mathrm{a}}$ and $W^{\mathrm{ab}}$. The R-symmetry group of the $\mathcal{N}=4$ superspace is $\mathrm{SO}(4) \cong\left(\mathrm{SU}(2)_{\mathrm{L}} \times \mathrm{SU}(2)_{\mathrm{R}}\right) / \mathbb{Z}_{2}$. This suggests that the $\mathrm{USp}(4)$ index 'a' splits into a pair of $\mathrm{SU}(2)$ indices $i$ and $\tilde{i}$,

$$
\Phi^{\mathrm{a}} \rightarrow\left(q^{i}, q^{\tilde{i}}\right) .
$$

Here $q^{i}$ and $q^{\tilde{i}}$ are left and right hypermultiplets, correspondingly. The $\mathcal{N}=5$ gauge superfield strength $W^{\text {ab }}$ has the following four $\mathcal{N}=4$ superfield components:

$$
W^{\mathrm{ab}} \rightarrow\left(W^{i j}, W^{i \tilde{j}}, W^{\tilde{i j}}, W^{\tilde{i \tilde{j}}}\right) .
$$

Here the superfields $W^{i j}$ and $W^{\tilde{i} \tilde{j}}$ constitute the field strength of the large $\mathcal{N}=4$ vector multiplet [25].

It is not hard to check that the $\mathcal{N}=5$ equation (2.37) leads to the standard hypermultiplet equations of motion

$$
\nabla_{\alpha}^{\tilde{i}(j} q^{k)}=0, \quad \nabla_{\alpha}^{i(\tilde{j}} q^{\tilde{k})}=0 .
$$

The $\mathcal{N}=5$ Bianchi identity (2.36) leads to the Bianchi identities for the $\mathcal{N}=4$ large vector multiplet

$$
\nabla_{\alpha}^{\tilde{i}(i} W^{j k)}=0, \quad \nabla_{\alpha}^{i(\tilde{i}} W^{\tilde{j} \tilde{k})}=0 .
$$

Reducing the equations (2.39) to the $\mathcal{N}=4$ superspace gives

$$
\begin{array}{ll}
W_{A}^{i j}=\mathrm{i} \kappa g_{A B} \bar{q}^{(i} T^{B} q^{j)}, & W_{A}^{\tilde{i} \tilde{j}}=\mathrm{i} \kappa g_{A B} \bar{q}^{(\tilde{i}} T^{B} q^{\tilde{j})}, \\
W_{A}^{i \tilde{j}}=\mathrm{i} \kappa g_{A B} \bar{q}^{(i} T^{B} q^{\tilde{j})}, & W_{A}^{\tilde{i} j}=\mathrm{i} \kappa g_{A B} \bar{q}^{(\tilde{i}} T^{B} q^{j)} .
\end{array}
$$

The equations obtained coincide with the $\mathcal{N}=4$ superfield equations of motion in the ABJM theory, which were given in [25].

The relation (2.44) has the following important consequence: every $\mathcal{N}=5$ superconformal field theory contains the same number of the left and right $\mathcal{N}=4$ hypermultiplets transforming in the same representation of the gauge group. This explains the vanishing of the coefficient $\tilde{d}_{\mathcal{N}=4}$ in $(2.21)$ for those $\mathcal{N}=4$ superconformal theories which possess $\mathcal{N}=5$ extended supersymmetry.

\section{$3 \mathcal{N}=6$ superconformal field theories}

Three-dimensional $\mathcal{N}=6$ superconformal field theories play an important role in the $\mathrm{AdS}_{4} / \mathrm{CFT}_{3}$ correspondence which has been intensively studied starting from the works $[4,5]$. The equations of motion of such theories in $\mathcal{N}=6$ superspace were studied in [18]. In this section we will compute the two- and three-point correlation functions of the supercurrent in these models and study their reduction to the $\mathcal{N}=5$ and $\mathcal{N}=4$ superspaces. 


\subsection{Correlators of $\mathcal{N}=6$ supercurrent}

As discussed in section 1 , the $\mathcal{N}=6$ supercurrent is a primary dimension- 1 superfield $J^{I J}=-J^{J I}$ subject to the conservation equation (1.5). The two-point function which is compatible with this conservation law is given by

$$
\left\langle J^{I J}\left(z_{1}\right) J^{K L}\left(z_{2}\right)\right\rangle=c_{\mathcal{N}=6} \frac{u_{12}^{I K} u_{12}^{J L}-u_{12}^{I L} u_{12}^{J K}}{\boldsymbol{x}_{12}{ }^{2}}
$$

where $c_{\mathcal{N}}=6$ is a free coefficient.

For the three-point correlator we make the standard ansatz

$$
\left\langle J^{I J}\left(z_{1}\right) J^{K L}\left(z_{2}\right) J^{M N}\left(z_{3}\right)\right\rangle=\frac{u_{13}^{I I^{\prime}} u_{13}^{J J^{\prime}} u_{23}^{K K^{\prime}} u_{23}^{L L^{\prime}}}{\boldsymbol{x}_{13}{ }^{2} \boldsymbol{x}_{23}{ }^{2}} H^{I^{\prime} J^{\prime} K^{\prime} L^{\prime} M N}\left(\boldsymbol{X}_{3}, \Theta_{3}\right),
$$

where the tensor $H^{I J K L M N}=H^{[I J][K L][M N]}$ obeys the equation

$$
\begin{aligned}
\frac{2}{3} \mathcal{D}_{\alpha}^{P} H^{I J K L M N}+\frac{1}{5} \mathcal{D}_{\alpha}^{Q}\left(H^{Q I K L M N} \delta^{J P}-H^{Q J K L M N} \delta^{I P}\right) & \\
& -\frac{1}{3}\left(\mathcal{D}_{\alpha}^{I} H^{J P K L M N}+\mathcal{D}_{\alpha}^{J} H^{P I K L M N}\right)=0,
\end{aligned}
$$

which is a consequence of (1.5). It has the scaling property similar to (2.3)

$$
H^{I J K L M N}\left(\lambda^{2} \boldsymbol{X}, \lambda \Theta\right)=\lambda^{-2} H^{I J K L M N}(\boldsymbol{X}, \Theta),
$$

and obeys the equations

$$
\begin{aligned}
H^{I J K L M N}(\boldsymbol{X}, \Theta)= & H^{K L I J M N}\left(-\boldsymbol{X}^{\mathrm{T}},-\Theta\right), \\
H^{I J K L M N}\left(-\boldsymbol{X}_{1}^{\mathrm{T}},-\Theta_{1}\right)= & \boldsymbol{x}_{13}{ }^{2} \boldsymbol{X}_{3}{ }^{2} u_{13}^{I I^{\prime}} u_{13}^{J J^{\prime}} u_{13}^{K K^{\prime}} u_{13}^{L L^{\prime}} u_{13}^{M M^{\prime}} u_{13}^{N N^{\prime}} U_{3}^{K^{\prime} P} U_{3}^{L^{\prime} R} \\
& \times H^{M^{\prime} N^{\prime} P R I^{\prime} J^{\prime}}\left(\boldsymbol{X}_{3}, \Theta_{3}\right),
\end{aligned}
$$

which follow from the invariance of the correlation function (3.2) under interchange of the order of operators.

We look for a solution of the above equations for the tensor $H^{I J K L M N}$ in the following form

$$
H^{I J K L M N}=\sum_{n} c_{n} H_{n}^{I J K L M N}
$$


where $c_{n}$ are some coefficients and

$$
\begin{aligned}
& H_{1}^{I J K L M N}=\frac{\varepsilon^{I J K L M N}}{X}, \\
& H_{2}^{I J K L M N}=\frac{A^{P Q}}{X^{3}}\left[\varepsilon^{J K M N P Q} \delta^{I L}+\varepsilon^{I L M N P Q} \delta^{J K}-\varepsilon^{I K M N P Q} \delta^{J L}-\varepsilon^{J L M N P Q} \delta^{I K}\right], \\
& H_{3}^{I J K L M N}=\frac{A^{P Q}}{X^{3}}\left[\varepsilon^{I J K M P Q} \delta^{L N}-\varepsilon^{I J L M P Q} \delta^{K N}-\varepsilon^{I J K N P Q} \delta^{L M}+\varepsilon^{I J L N P Q} \delta^{K M}\right], \\
& H_{4}^{I J K L M N}=\frac{A^{P Q}}{X^{3}}\left[\varepsilon^{K L J M P Q} \delta^{I N}+\varepsilon^{K L I N P Q} \delta^{J M}-\varepsilon^{K L I M P Q} \delta^{J N}-\varepsilon^{K L J N P Q} \delta^{I M}\right] \\
& H_{5}^{I J K L M N}=\frac{A^{P Q} A^{R S}}{X^{5}}\left[\varepsilon^{M N P Q R S}\left(\delta^{J K} \delta^{I L}-\delta^{I K} \delta^{J L}\right)\right], \\
& H_{6}^{I J K L M N}=\frac{A^{P Q} A^{R S}}{X^{5}}\left[\varepsilon^{I J P Q R S}\left(\delta^{L M} \delta^{K N}-\delta^{K M} \delta^{L N}\right)\right], \\
& H_{7}^{I J K L M N}=\frac{A^{P Q} A^{R S}}{X^{5}}\left[\varepsilon^{K L P Q R S}\left(\delta^{J M} \delta^{I N}-\delta^{I M} \delta^{J N}\right)\right], \\
& H_{8}^{I J K L M N}=\frac{A^{P Q} A^{R S}}{X^{5}}\left[\varepsilon^{J K P Q R S}\left(\delta^{I M} \delta^{L N}-\delta^{I N} \delta^{L M}\right)-\varepsilon^{I K P Q R S}\left(\delta^{J M} \delta^{L N}-\delta^{J N} \delta^{L M}\right)\right. \\
& \left.-\varepsilon^{J L P Q R S}\left(\delta^{I M} \delta^{K N}-\delta^{I N} \delta^{K M}\right)+\varepsilon^{I L P Q R S}\left(\delta^{J M} \delta^{K N}-\delta^{J N} \delta^{K M}\right)\right], \\
& H_{9}^{I J K L M N}=\frac{A^{P Q} A^{R S}}{X^{5}}\left[\varepsilon^{I M P Q R S}\left(\delta^{J K} \delta^{L N}-\delta^{J L} \delta^{K N}\right)-\varepsilon^{J M P Q R S}\left(\delta^{I K} \delta^{L N}-\delta^{I L} \delta^{K N}\right)\right. \\
& \left.-\varepsilon^{I N P Q R S}\left(\delta^{J K} \delta^{L M}-\delta^{J L} \delta^{K M}\right)+\varepsilon^{J N P Q R S}\left(\delta^{I K} \delta^{L M}-\delta^{I L} \delta^{K M}\right)\right], \\
& H_{10}^{I J K L M N}=\frac{A^{P Q} A^{R S}}{X^{5}}\left[\varepsilon^{K M P Q R S}\left(\delta^{I L} \delta^{J N}-\delta^{J L} \delta^{I N}\right)-\varepsilon^{L M P Q R S}\left(\delta^{I K} \delta^{J N}-\delta^{J K} \delta^{I N}\right)\right. \\
& \left.-\varepsilon^{K N P Q R S}\left(\delta^{I L} \delta^{J M}-\delta^{J L} \delta^{I M}\right)+\varepsilon^{L N P Q R S}\left(\delta^{I K} \delta^{J M}-\delta^{J K} \delta^{I M}\right)\right], \\
& H_{11}^{I J K L M N}=\frac{\varepsilon^{P Q R S T U} A^{P Q} A^{R S} A^{T U}}{X^{7}}\left[\delta^{J K}\left(\delta^{I M} \delta^{L N}-\delta^{I N} \delta^{L M}\right)-\delta^{I K}\left(\delta^{J M} \delta^{L N}-\delta^{J N} \delta^{L M}\right)\right. \\
& \left.+\delta^{I L}\left(\delta^{J M} \delta^{K N}-\delta^{J N} \delta^{K M}\right)-\delta^{J L}\left(\delta^{I M} \delta^{K N}-\delta^{I N} \delta^{K M}\right)\right] .
\end{aligned}
$$

The tensors (3.8) obey the constraints (3.4) and (3.5) by construction. Imposing the equation (3.3) we find the coefficients $c_{n}$

$$
\begin{aligned}
& c_{1}=2 d_{\mathcal{N}=6}, \quad c_{2}=c_{3}=c_{4}=d_{\mathcal{N}=6}, \quad c_{11}=\frac{1}{24} d_{\mathcal{N}=6}, \\
& c_{5}=c_{6}=c_{7}=c_{8}=c_{9}=c_{10}=\frac{1}{4} d_{\mathcal{N}=6}
\end{aligned}
$$

where $d_{\mathcal{N}=6}$ is a free coefficient. It is possible to show that for these values of the coefficients the tensor (3.7) obeys the equation (3.6) which ensures the invariance of the correlation function under the interchange of operators $J^{I J}\left(z_{1}\right)$ and $J^{M N}\left(z_{3}\right)$. However, it is a tedious exercise to demonstrate this directly. Instead of embarking on such an exercise, we will take a shortcut and prove the required symmetry property using the $\mathcal{N}=6 \rightarrow \mathcal{N}=5$ superspace reduction of the superfield operators and their correlation functions. 


\section{$3.2 \mathcal{N}=6 \rightarrow \mathcal{N}=5$ superspace reduction}

Let us split the $\mathrm{SO}(6)$ index $I$ as $I=(\hat{I}, 6), \hat{I}=1,2,3,4,5$. Upon reduction to $\mathcal{N}=5$ superspace, the $\mathcal{N}=6$ supercurrent $J^{I J}$ leads to two $\mathcal{N}=5$ superfields, one of which is the $\mathcal{N}=5$ supercurrent $J^{\hat{I}}$ and the other is an antisymmetric tensor $K^{\hat{I} \hat{J}}$ :

$$
J^{\hat{I}}=J^{\hat{I} 6}\left|, \quad K^{\hat{I} \hat{J}}=J^{\hat{I} \hat{J}}\right|
$$

where the bar-projection means setting $\theta_{\alpha}^{6}=0$. As a consequence of the $\mathcal{N}=6$ supercurrent conservation equation (1.5), $J^{\hat{I}}$ proves to obey the $\mathcal{N}=5$ supercurrent conservation equation (1.3), while for $K^{\hat{I} \hat{J}}$ we obtain the following constraint

$$
D_{\alpha}^{\hat{I}} K^{\hat{J} \hat{K}}=D_{\alpha}^{[\hat{I}} K^{\hat{J} \hat{K}]}-\frac{1}{2} D_{\alpha}^{\hat{L}} K^{\hat{L}[\hat{J}} \delta^{\hat{K}] \hat{I}}
$$

The $\mathcal{N}=6 \rightarrow \mathcal{N}=5$ superspace reduction of the two-point structure $u_{12}^{I J}$ is similar to (2.15). Therefore, the reduction of the two-point correlation function (3.1) is rather trivial

$$
\begin{aligned}
\left\langle K^{\hat{I} \hat{J}}\left(z_{1}\right) K^{\hat{K} \hat{L}}\left(z_{2}\right)\right\rangle & =c_{\mathcal{N}}=6 \frac{u_{12}^{\hat{I} \hat{K}} u_{12}^{\hat{J} \hat{L}}-u_{12}^{\hat{I} \hat{L}} u_{12}^{\hat{J} \hat{K}}}{\boldsymbol{x}_{12}{ }^{2}} \\
\left\langle J^{\hat{I}}\left(z_{1}\right) J^{\hat{J}}\left(z_{2}\right)\right\rangle & =c_{\mathcal{N}}=6 \frac{u_{12}^{\hat{I} \hat{J}}}{\boldsymbol{x}_{12}{ }^{2}} .
\end{aligned}
$$

The three-point function (3.2) reduces to the following four correlation functions in the $\mathcal{N}=5$ superspace

$$
\left\langle K^{\hat{I} \hat{J}} K^{\hat{K} \hat{L}} K^{\hat{M} \hat{N}}\right\rangle, \quad\left\langle K^{\hat{I} \hat{J}} K^{\hat{K} \hat{L}} J^{\hat{M}}\right\rangle, \quad\left\langle K^{\hat{I} \hat{J}} J^{\hat{K}} J^{\hat{L}}\right\rangle, \quad\left\langle J^{\hat{I}} J^{\hat{J}} J^{\hat{K}}\right\rangle .
$$

These correlators can be obtained by considering different components of the tensor (3.7). In particular, from the explicit form of the tensors (3.8) we immediately see that two of the four correlators vanish

$$
\left\langle K^{\hat{I} \hat{J}} K^{\hat{K} \hat{L}} K^{\hat{M} \hat{N}}\right\rangle=0, \quad\left\langle K^{\hat{I} \hat{J}} J^{\hat{K}} J^{\hat{L}}\right\rangle=0
$$

For the other two we find

$$
\begin{aligned}
\left\langle J^{\hat{I}}\left(z_{1}\right) J^{\hat{J}}\left(z_{2}\right) J^{\hat{K}}\left(z_{3}\right)\right\rangle= & \frac{u_{13}^{\hat{I}^{\prime}} u_{23}^{\hat{J} \hat{J}^{\prime}}}{\boldsymbol{x}_{13}{ }^{2} \boldsymbol{x}_{23}{ }^{2}} H_{\mathcal{N}=5}^{\hat{I}^{\prime} \hat{J}^{\prime} \hat{K}}\left(\boldsymbol{X}_{3}, \Theta_{3}\right) \\
H_{\mathcal{N}=5}^{\hat{I} \hat{J} \hat{K}}=H^{\hat{I} 6 \hat{J} 6 \hat{K} 6} \mid= & d_{\mathcal{N}=6} \frac{1}{4}\left(\delta^{\hat{I} \hat{J}} \varepsilon^{\hat{K} \hat{L} \hat{M} \hat{N} \hat{P}}-\delta^{\hat{I} \hat{K}} \varepsilon^{\hat{J} \hat{L} \hat{M} \hat{N} \hat{P}}-\delta^{\hat{J} \hat{K}} \varepsilon^{\hat{I} \hat{L} \hat{M} \hat{N} \hat{P}}\right) \frac{A^{\hat{L} \hat{M}} A^{\hat{N} \hat{P}}}{X^{5}} \\
& +d_{\mathcal{N}=6} \varepsilon^{\hat{I} \hat{J} \hat{K} \hat{L} \hat{M}} \frac{A^{\hat{L} \hat{M}}}{X^{3}}
\end{aligned}
$$


and

$$
\begin{aligned}
& \left\langle K^{\hat{I} \hat{J}}\left(z_{1}\right) J^{\hat{K}}\left(z_{2}\right) K^{\hat{M} \hat{N}}\left(z_{3}\right)\right\rangle=\frac{u_{13}^{\hat{I} \hat{I}^{\prime}} u_{13}^{\hat{J} \hat{J}^{\prime}} u_{23}^{\hat{K} \hat{K}^{\prime}}}{\boldsymbol{x}_{13}{ }^{2} \boldsymbol{x}_{23}{ }^{2}} H_{\mathcal{N}=5}^{\hat{I}^{\prime} \hat{J}^{\prime} \hat{K}^{\prime} \hat{M} \hat{N}}\left(\boldsymbol{X}_{3}, \Theta_{3}\right), \\
& H_{\mathcal{N}=5}^{\hat{I} \hat{J} \hat{K} \hat{M} \hat{N}}=H^{\hat{I} \hat{J} \hat{K} 6 \hat{M} \hat{N}} \mid=2 d_{\mathcal{N}=6} \frac{\varepsilon^{\hat{I} \hat{J} \hat{K} \hat{M} \hat{N}}}{X} \\
& +d_{\mathcal{N}=6} \frac{A^{\hat{P} \hat{Q}}}{X^{3}}\left[\varepsilon^{\hat{I} \hat{M} \hat{N} \hat{P} \hat{Q}} \delta^{\hat{J} \hat{K}}-\varepsilon^{\hat{J} \hat{M} \hat{N} \hat{P} \hat{Q}} \delta^{\hat{I} \hat{K}}-\varepsilon^{\hat{I} \hat{J} \hat{N} \hat{P}} \hat{Q} \delta^{\hat{K} \hat{M}}\right. \\
& \left.+\varepsilon^{\hat{I} \hat{J} \hat{M} \hat{P} \hat{Q}} \delta^{\hat{K} \hat{N}}-\varepsilon^{\hat{K} \hat{I} \hat{M} \hat{P} \hat{Q}} \delta^{\hat{J} \hat{N}}+\varepsilon^{\hat{K} \hat{J} \hat{M} \hat{P} \hat{Q}} \delta^{\hat{I} \hat{N}}+\varepsilon^{\hat{K} \hat{I} \hat{N} \hat{P} \hat{Q}} \delta^{\hat{J} \hat{M}}-\varepsilon^{\hat{K} \hat{J} \hat{N} \hat{P} \hat{Q}} \delta^{\hat{I} \hat{M}}\right] \\
& +\frac{1}{4} d_{\mathcal{N}=6} \frac{A^{\hat{P} \hat{Q}} A^{\hat{R} \hat{S}}}{X^{5}}\left[\varepsilon^{\hat{K} \hat{P} \hat{Q} \hat{R} \hat{S}}\left(\delta^{\hat{J} \hat{M}} \delta^{\hat{I} \hat{N}}-\delta^{\hat{I} \hat{M}} \delta^{\hat{J} \hat{N}}\right)-\varepsilon^{\hat{J} \hat{P} \hat{Q} \hat{R} \hat{S}}\left(\delta^{\hat{I} \hat{M}} \delta^{\hat{K} \hat{N}}-\delta^{\hat{K} \hat{M}} \delta^{\hat{I} \hat{N}}\right)\right. \\
& +\varepsilon^{\hat{I} \hat{P} \hat{Q} \hat{R} \hat{S}}\left(\delta^{\hat{J} \hat{M}} \delta^{\hat{K} \hat{N}}-\delta^{\hat{J} \hat{N}} \delta^{\hat{K} \hat{M}}\right)+\varepsilon^{\hat{M} \hat{P} \hat{Q} \hat{R} \hat{S}}\left(\delta^{\hat{I} \hat{K}} \delta^{\hat{J} \hat{N}}-\delta^{\hat{J} \hat{K}} \delta^{\hat{I} \hat{N}}\right) \\
& \left.-\varepsilon^{\hat{N} \hat{P} \hat{Q} \hat{R} \hat{S}}\left(\delta^{\hat{I} \hat{K}} \delta^{\hat{J} \hat{M}}-\delta^{\hat{J} \hat{K}} \delta^{\hat{I} \hat{M}}\right)\right] \text {. }
\end{aligned}
$$

Comparing (3.16b) with (2.7) we find the relation among the coefficients

$$
d_{\mathcal{N}=6}=4 d_{\mathcal{N}=5} .
$$

This relation, in conjunction with (2.24), gives us the ratio between the coefficients of twoand three-point functions

$$
\frac{d_{\mathcal{N}=6}}{c_{\mathcal{N}=6}}=4 \frac{d_{\mathcal{N}=5}}{c_{\mathcal{N}=5}}=\frac{1}{4 \pi} .
$$

This relation is a manifestation of a Ward identity which relates the supercurrent two-point and three-point functions.

The tensor $(3.17 \mathrm{~b})$ can be represented in the equivalent form

$$
H_{\mathcal{N}=5}^{\hat{I} \hat{J} \hat{K} \hat{L} \hat{M}}=d_{\mathcal{N}=6} \sum_{n} a_{n} H_{n}^{\hat{I} \hat{J} \hat{K} \hat{L} \hat{M}}
$$

where the coefficients $a_{n}$ are

$$
\begin{aligned}
& a_{1}=a_{2}=1, \quad a_{3}=a_{4}=-\frac{1}{2}, \quad a_{5}=a_{6}=a_{7}=a_{8}=\frac{1}{2}, \quad a_{9}=-\frac{1}{4}, \\
& a_{10}=a_{11}=a_{12}=a_{13}=a_{14}=a_{15}=\frac{1}{8}, \quad a_{16}=-\frac{3}{8}, \quad a_{17}=\frac{3}{64},
\end{aligned}
$$


and the tensors $H_{n}^{\hat{I} \hat{J} \hat{K} \hat{L} \hat{M}}$ are expressed in terms of the covariant objects (A.8)

$$
\begin{aligned}
& H_{1}^{\hat{I} \hat{J} \hat{K} \hat{M} \hat{N}}=\frac{\varepsilon^{\hat{I} \hat{J} \hat{K} \hat{M} \hat{N}}}{\boldsymbol{X}}, \\
& H_{2}^{\hat{I} \hat{J} \hat{K} \hat{M} \hat{N}}=\frac{U^{\hat{K}^{\prime} \hat{K}} \varepsilon^{\hat{I} \hat{J} \hat{K}^{\prime} \hat{M} \hat{N}}}{\boldsymbol{X}}, \\
& H_{3}^{\hat{I} \hat{J} \hat{K} \hat{M} \hat{N}}=-\frac{1}{2} \frac{U^{\hat{P} \hat{Q}}}{\boldsymbol{X}}\left(\varepsilon^{\hat{J} \hat{M} \hat{N} \hat{P} \hat{Q}} \delta^{\hat{I} \hat{K}}-\varepsilon^{\hat{I} \hat{M} \hat{N} \hat{P}} \hat{Q} \delta^{\hat{J} \hat{K}}\right), \\
& H_{4}^{\hat{I} \hat{J} \hat{K} \hat{M} \hat{N}}=\frac{1}{2} \frac{U^{\hat{P} \hat{Q}}}{\boldsymbol{X}}\left(U^{\hat{M} \hat{K}} \varepsilon^{\hat{N} \hat{I} \hat{J} \hat{P} \hat{Q}}-U^{\hat{N} \hat{K}} \varepsilon^{\hat{M} \hat{I} \hat{J} \hat{P} \hat{Q}}\right), \\
& H_{5}^{\hat{I} \hat{J} \hat{K} \hat{M} \hat{N}}=-\frac{1}{2} \frac{U^{\hat{P} \hat{Q}}}{\boldsymbol{X}}\left(\varepsilon^{\hat{I} \hat{J} \hat{M} \hat{P} \hat{Q}} \delta^{\hat{K} \hat{N}}-\varepsilon^{\hat{I} \hat{J} \hat{N} \hat{P} \hat{Q}} \delta^{\hat{K} \hat{M}}\right), \\
& H_{6}^{\hat{I} \hat{J} \hat{K} \hat{M} \hat{N}}=\frac{1}{2} \frac{U^{\hat{P} \hat{Q}}}{\boldsymbol{X}}\left(\varepsilon^{\hat{K} \hat{I} \hat{M} \hat{P} \hat{Q}} \delta^{\hat{J} \hat{N}}-\varepsilon^{\hat{K} \hat{J} \hat{M} \hat{P} \hat{Q}} \delta^{\hat{I} \hat{N}}-\varepsilon^{\hat{K} \hat{I} \hat{N} \hat{P} \hat{Q}} \delta^{\hat{J} \hat{M}}+\varepsilon^{\hat{K} \hat{J} \hat{N} \hat{P} \hat{Q}} \delta^{\hat{I} \hat{M}}\right) \\
& H_{7}^{\hat{I} \hat{J} \hat{K} \hat{M} \hat{N}}=\frac{1}{2} \frac{U^{\hat{P} \hat{Q}}}{\boldsymbol{X}}\left(U^{\hat{J} \hat{K}} \varepsilon^{\hat{M} \hat{N} \hat{I} \hat{P} \hat{Q}}-U^{\hat{I} \hat{K}} \varepsilon^{\hat{M} \hat{N} \hat{J} \hat{P} \hat{Q}}\right), \\
& H_{8}^{\hat{I} \hat{J} \hat{K} \hat{M} \hat{N}}=\frac{1}{2} \frac{U^{\hat{P} \hat{Q}} U^{\hat{L} \hat{K}}}{\boldsymbol{X}}\left(\delta^{\hat{J} \hat{M}} \varepsilon^{\hat{L} \hat{N} \hat{I} \hat{P} \hat{Q}}+\delta^{\hat{I} \hat{N}} \varepsilon^{\hat{L} \hat{M} \hat{J} \hat{P} \hat{Q}}-\delta^{\hat{J} \hat{N}} \varepsilon^{\hat{L} \hat{M} \hat{I} \hat{P} \hat{Q}}-\delta^{\hat{I} \hat{M}} \varepsilon^{\hat{L} \hat{N} \hat{J} \hat{P} \hat{Q}}\right), \\
& H_{9}^{\hat{I} \hat{J} \hat{K} \hat{M} \hat{N}}=\frac{1}{4} \frac{U^{\hat{P} \hat{Q}} U^{\hat{R} \hat{S}}}{\boldsymbol{X}} \varepsilon^{\hat{K} \hat{P} \hat{Q} \hat{R} \hat{S}}\left(\delta^{\hat{I} \hat{M}} \delta^{\hat{J} \hat{N}}-\delta^{\hat{J} \hat{M}} \delta^{\hat{I} \hat{N}}\right), \\
& H_{10}^{\hat{I} \hat{J} \hat{K} \hat{M} \hat{N}}=\frac{1}{4} \frac{U^{\hat{P} \hat{Q}} U^{\hat{R} \hat{S}}}{\boldsymbol{X}}\left[\varepsilon^{\hat{I} \hat{P} \hat{Q} \hat{R} \hat{S}}\left(\delta^{\hat{J} \hat{M}} \delta^{\hat{K} \hat{N}}-\delta^{\hat{J} \hat{N}} \delta^{\hat{K} \hat{M}}\right)-(\hat{I} \leftrightarrow \hat{J})\right] \\
& H_{11}^{\hat{I} \hat{J} \hat{K} \hat{M} \hat{N}}=\frac{1}{4} \frac{U^{\hat{P} \hat{Q}} U^{\hat{R} \hat{S}}}{\boldsymbol{X}}\left[\varepsilon^{\hat{M} \hat{P} \hat{Q} \hat{R} \hat{S}}\left(\delta^{\hat{I} \hat{N}} U^{\hat{J} \hat{K}}-\delta^{\hat{J} \hat{N}} U^{\hat{I} \hat{K}}\right)-(\hat{M} \leftrightarrow \hat{N})\right], \\
& H_{12}^{\hat{I} \hat{J} \hat{K} \hat{M} \hat{N}}=\frac{1}{4} \frac{U^{\hat{P} \hat{Q}} U^{\hat{R} \hat{S}}}{\boldsymbol{X}}\left[\varepsilon^{\hat{M} \hat{P} \hat{Q} \hat{R} \hat{S}}\left(\delta^{\hat{I} \hat{K}} \delta^{\hat{J} \hat{N}}-\delta^{\hat{J} \hat{K}} \delta^{\hat{I} \hat{N}}\right)-(\hat{M} \leftrightarrow \hat{N})\right], \\
& H_{13}^{\hat{I} \hat{J} \hat{K} \hat{M} \hat{N}}=\frac{1}{4} \frac{U^{\hat{P} \hat{Q}} U^{\hat{R} \hat{S}}}{\boldsymbol{X}}\left[\varepsilon^{\hat{J} \hat{P} \hat{Q} \hat{R} \hat{S}}\left(\delta^{\hat{I} \hat{M}} U^{\hat{N} \hat{K}}-\delta^{\hat{I} \hat{N}} U^{\hat{M} \hat{K}}\right)-(\hat{I} \leftrightarrow \hat{J})\right], \\
& H_{14}^{\hat{I} \hat{J} \hat{K} \hat{M} \hat{N}}=\varepsilon^{\hat{I} \hat{J} \hat{K} \hat{M} \hat{N}} \frac{\Theta^{4}}{\boldsymbol{X}^{3}}, \\
& H_{15}^{\hat{I} \hat{J} \hat{K} \hat{M} \hat{N}}=\frac{U^{\hat{K}^{\prime} \hat{K}} \varepsilon^{\hat{I} \hat{J} \hat{K}^{\prime} \hat{M} \hat{N}} \Theta^{4}}{\boldsymbol{X}^{3}}, \\
& H_{16}^{\hat{I} \hat{J} \hat{K} \hat{M} \hat{N}}=-\frac{1}{2} \frac{\Theta^{4}}{\boldsymbol{X}^{3}} U^{\hat{P} \hat{Q}}\left(\varepsilon^{\hat{K} \hat{I} \hat{M} \hat{P} \hat{Q}} \delta^{\hat{J} \hat{N}}-\varepsilon^{\hat{K} \hat{J} \hat{M} \hat{P} \hat{Q}} \delta^{\hat{I} \hat{N}}-\varepsilon^{\hat{K} \hat{I} \hat{N} \hat{P} \hat{Q}} \delta^{\hat{J} \hat{M}}+\varepsilon^{\hat{K} \hat{J} \hat{N} \hat{P} \hat{Q}} \delta^{\hat{I} \hat{M}}\right) \\
& H_{17}^{\hat{I} \hat{J} \hat{K} \hat{M} \hat{N}}=\frac{\Theta^{8}}{\boldsymbol{X}^{5}} \varepsilon^{\hat{I} \hat{J} \hat{K} \hat{M} \hat{N}} .
\end{aligned}
$$

In verifying that (3.20) coincides with $(3.17 \mathrm{~b})$, it is advantageous to use the rela- 
tions (2.10)-(2.12) as well as the following $\mathcal{N}=5$ superspace identities

$$
\begin{aligned}
A^{I J} A^{K L}= & A^{[I J} A^{K L]}-\frac{1}{3} X^{2}\left(B^{I K} B^{J L}-B^{J K} B^{I L}\right), \\
\frac{4}{3} B^{Q K}\left(B^{P I} \varepsilon^{M N J P Q}-B^{P J} \varepsilon^{M N I P Q}\right)= & \frac{4}{3} B^{P K}\left(B^{Q M} \varepsilon^{I J N P Q}-B^{Q N} \varepsilon^{I J M P Q}\right) \\
& -2 \Theta^{2} B^{P K} \varepsilon^{I J M N P} \\
A^{P Q}\left(B^{I K} \varepsilon^{M N J P Q}-B^{J K} \varepsilon^{M N I P Q}\right)= & A^{P Q}\left(B^{N K} \varepsilon^{I J P Q M}-B^{M K} \varepsilon^{I J N P Q}\right) \\
& -\Theta^{2} A^{P K} \varepsilon^{I J M N P}
\end{aligned}
$$

where $B^{I J} \equiv \Theta^{I \alpha} \Theta_{\alpha}^{J}$.

For the tensor $H^{\hat{I} \hat{J} \hat{K} \hat{M} \hat{N}}$ in the form (3.20) it is possible to check that it obeys the equation

$$
H^{\hat{I} \hat{J} \hat{K} \hat{M} \hat{N}}\left(-\boldsymbol{X}_{1}^{\mathrm{T}},-\Theta_{1}\right)=\boldsymbol{x}_{13}{ }^{2} \boldsymbol{X}_{3}{ }^{2} u_{13}^{\hat{I} \hat{I}^{\prime}} u_{13}^{\hat{J} \hat{J}^{\prime}} u_{13}^{\hat{K} \hat{K}^{\prime}} u_{13}^{\hat{M} \hat{M}^{\prime}} u_{13}^{\hat{N} \hat{N}^{\prime}} U_{3}^{\hat{K}^{\prime} \hat{P}} H^{\hat{M}^{\prime} \hat{N}^{\prime} \hat{P} \hat{I}^{\prime} \hat{J}^{\prime}}\left(\boldsymbol{X}_{3}, \Theta_{3}\right),
$$

which ensures the invariance of the correlation function (3.17) under the interchange of operators $K^{\hat{I} \hat{J}}\left(z_{1}\right)$ and $K^{\hat{M} \hat{N}}\left(z_{3}\right)$. Thus, the two $\mathcal{N}=5$ projections (3.16) and (3.17) are invariant under interchange of positions of operators. This proves that the $\mathcal{N}=6$ correlation function (3.2) also respects this symmetry.

\subsection{Further $\mathcal{N}=5 \rightarrow \mathcal{N}=4$ superspace reduction}

The $\mathcal{N}=6$ correlator (3.2) reduces to two $\mathcal{N}=5$ correlation functions (3.16) and (3.17), one of which is just the $\mathcal{N}=5$ supercurrent correlator while the other is the mixed correlator. The reduction to the $\mathcal{N}=4$ superspace of the $\mathcal{N}=5$ supercurrent correlation function was considered in section 2.2. Here we will study the $\mathcal{N}=4$ superspace reduction of the mixed correlator (3.17).

In this subsection the $\mathrm{SO}(5)$ indices are denoted by $I, J, K, \ldots$, while the $\mathrm{SO}(4)$ indices are denoted by the same letters with hats, e.g., $\hat{I}, \hat{J}, \hat{K}, \ldots$ The $\mathcal{N}=4$ superspace components of the $\mathcal{N}=5$ supercurrent are given in (2.13). We define the $\mathcal{N}=4$ superspace projections of the antisymmetric tensor $K^{I J}$ as

$$
L^{\hat{I} \hat{J}}=K^{\hat{I} \hat{J}}\left|, \quad R^{\hat{I}}=K^{\hat{I} 5}\right|,
$$

where the bar-projection means $\theta_{\alpha}^{5}=0$. As a consequence of (3.12) they obey the following equations

$$
\begin{aligned}
& D_{\alpha}^{\hat{I}} L^{\hat{J} \hat{K}}=D_{\alpha}^{[\hat{I}} L^{\hat{J} \hat{K}]}-\frac{2}{3} D_{\alpha}^{\hat{L}} L^{\hat{L}[\hat{J}} \delta^{\hat{K}] \hat{I}} \\
& D_{\alpha}^{(\hat{I}} R^{\hat{J})}-\frac{1}{4} \delta^{\hat{I} \hat{J}} D_{\alpha}^{\hat{K}} R^{\hat{K}}=0
\end{aligned}
$$

The latter equation coincides with (2.14a) while the former shows that $L^{\hat{I} \hat{J}}$ describes the $\mathcal{N}=4$ flavour current multiplets. The antisymmetric tensor $L^{\hat{I} \hat{J}}$ can be further decomposed into two components $L_{+}^{\hat{I} \hat{J}}$ and $L_{-}^{\hat{I} \hat{J}}$ with different self-duality properties

$$
\frac{1}{2} \varepsilon^{\hat{I} \hat{J} \hat{K} \hat{L}} L_{ \pm}^{\hat{K} \hat{L}}= \pm L_{ \pm}^{\hat{I} \hat{J}} .
$$


The correlator $\left\langle K^{I J} J^{K} K^{M N}\right\rangle$ reduces to the following six correlation functions of the $\mathcal{N}=4$ superfields

$$
\left\langle L^{\hat{I} \hat{J}} S^{\hat{K}} L^{\hat{M} \hat{N}}\right\rangle,\left\langle L^{\hat{I} \hat{J}} J L^{\hat{M} \hat{N}}\right\rangle, \quad\left\langle L^{\hat{I} \hat{J}} S^{\hat{K}} R^{\hat{M}}\right\rangle, \quad\left\langle L^{\hat{I} \hat{J}} J R^{\hat{M}}\right\rangle, \quad\left\langle R^{\hat{I}} S^{\hat{K}} R^{\hat{M}}\right\rangle, \quad\left\langle R^{\hat{I}} J R^{\hat{M}}\right\rangle .
$$

They can be found by taking $\mathcal{N}=4$ superspace projections of the tensor $(3.17 \mathrm{~b})$. In particular, it is easy to see that three of these six correlators vanish

$$
\left\langle L^{\hat{I} \hat{J}} S^{\hat{K}} L^{\hat{M} \hat{N}}\right\rangle=0, \quad\left\langle L^{\hat{I} \hat{J}} J R^{\hat{M}}\right\rangle=0, \quad\left\langle R^{\hat{I}} S^{\hat{K}} R^{\hat{M}}\right\rangle=0 .
$$

For the other three we find

$$
\begin{aligned}
& \left\langle L^{\hat{I} \hat{J}}\left(z_{1}\right) J\left(z_{2}\right) L^{\hat{M} \hat{N}}\left(z_{3}\right)\right\rangle=\frac{u_{13}^{\hat{I} \hat{I}^{\prime}} u_{13}^{\hat{J} \hat{J}^{\prime}}}{\boldsymbol{x}_{13}{ }^{2} \boldsymbol{x}_{23}{ }^{2}} H^{\hat{I}^{\prime} \hat{J}^{\prime} \hat{M} \hat{N}}\left(\boldsymbol{X}_{3}, \Theta_{3}\right), \\
& H^{\hat{I} \hat{J} \hat{M} \hat{N}}=d_{\mathcal{N}=6}\left[2 \frac{\varepsilon^{\hat{I} \hat{J} \hat{M} \hat{N}}}{X}+\frac{1}{4} \frac{A^{\hat{P} \hat{Q}} A^{\hat{R} \hat{S}}}{X^{5}} \varepsilon^{\hat{P} \hat{Q} \hat{R} \hat{S}}\left(\delta^{\hat{J} \hat{M}} \delta^{\hat{I} \hat{N}}-\delta^{\hat{I} \hat{M}} \delta^{\hat{J} \hat{N}}\right)\right. \\
& \left.+\frac{A^{\hat{P} \hat{Q}}}{X^{3}}\left(\varepsilon^{\hat{J} \hat{M} \hat{P} \hat{Q}} \delta^{\hat{I} \hat{N}}+\varepsilon^{\hat{I} \hat{N} \hat{P} \hat{Q}} \delta^{\hat{J} \hat{M}}-\varepsilon^{\hat{J} \hat{N} \hat{P} \hat{Q}} \delta^{\hat{I} \hat{M}}-\varepsilon^{\hat{I} \hat{M} \hat{P} \hat{Q}} \delta^{\hat{J} \hat{N}}\right)\right] \\
& \left\langle L^{\hat{I} \hat{J}}\left(z_{1}\right) S^{\hat{K}}\left(z_{2}\right) R^{\hat{L}}\left(z_{3}\right)\right\rangle=\frac{u_{13}^{\hat{I} \hat{I}^{\prime}} u_{13}^{\hat{J} \hat{J}^{\prime}} u_{23}^{\hat{K} \hat{K}^{\prime}}}{\boldsymbol{x}_{13}{ }^{2} \boldsymbol{x}_{23}{ }^{2}} H^{\hat{I}^{\prime} \hat{J}^{\prime} \hat{K}^{\prime} \hat{L}}\left(\boldsymbol{X}_{3}, \Theta_{3}\right), \\
& H^{\hat{I} \hat{J} \hat{K} \hat{L}}=d_{\mathcal{N}=6}\left[2 \frac{\varepsilon^{\hat{I} \hat{J} \hat{K} \hat{L}}}{X}+\frac{1}{4} \frac{A^{\hat{P} \hat{Q}} A^{\hat{R} \hat{S}}}{X^{5}} \varepsilon^{\hat{P} \hat{Q} \hat{R} \hat{S}}\left(\delta^{\hat{J} \hat{K}} \delta^{\hat{I} \hat{L}}-\delta^{\hat{I} \hat{K}} \delta^{\hat{J} \hat{L}}\right)\right. \\
& \left.+\frac{A^{\hat{P} \hat{Q}}}{X^{3}}\left(\varepsilon^{\hat{I} \hat{L} \hat{P} \hat{Q}} \delta^{\hat{J} \hat{K}}-\varepsilon^{\hat{J} \hat{L} \hat{P} \hat{Q}} \delta^{\hat{I} \hat{K}}-\varepsilon^{\hat{I} \hat{J} \hat{P} \hat{Q}} \delta^{\hat{K} \hat{L}}+\varepsilon^{\hat{K} \hat{I} \hat{P}} \hat{Q} \delta^{\hat{J} \hat{L}}-\varepsilon^{\hat{K} \hat{J} \hat{P} \hat{Q}} \delta^{\hat{I} \hat{L}}\right)\right] \\
& \left\langle R^{\hat{I}}\left(z_{1}\right) J\left(z_{2}\right) R^{\hat{J}}\left(z_{3}\right)\right\rangle=\frac{u_{13}^{\hat{I} \hat{I}^{\prime}}}{\boldsymbol{x}_{13}{ }^{2} \boldsymbol{x}_{23}{ }^{2}} H^{\hat{I}^{\prime} \hat{J}}\left(\boldsymbol{X}_{3}, \Theta_{3}\right), \\
& H^{\hat{I} \hat{J}}=-d_{\mathcal{N}=6}\left[\varepsilon^{\hat{I} \hat{J} \hat{K} \hat{L}} \frac{A^{\hat{K} \hat{L}}}{X^{3}}+\frac{1}{4} \delta^{\hat{I} \hat{J}} \varepsilon^{\hat{K} \hat{L} \hat{M} \hat{N}} \frac{A^{\hat{K} \hat{L}} A^{\hat{M} \hat{N}}}{X^{5}}\right] .
\end{aligned}
$$

The correlation function (3.31) can be decomposed into two parts with opposite selfduality properties

$$
\begin{aligned}
&\left\langle L_{ \pm}^{\hat{I} \hat{J}}\left(z_{1}\right) J\left(z_{2}\right) L_{ \pm}^{\hat{M} \hat{N}}\left(z_{3}\right)\right\rangle=\frac{u_{13}^{\hat{I} \hat{I}^{\prime}} u_{13}^{\hat{J} \hat{J}^{\prime}}}{\boldsymbol{x}_{13}{ }^{2} \boldsymbol{x}_{23}{ }^{2}} H_{ \pm}^{\hat{I}^{\prime} \hat{J}^{\prime} \hat{M} \hat{N}}\left(\boldsymbol{X}_{3}, \Theta_{3}\right) \\
& H_{ \pm}^{\hat{I} \hat{J} \hat{M} \hat{N}}= \frac{1}{2} H^{\hat{I} \hat{J} \hat{M} \hat{N}} \pm \frac{1}{4} \varepsilon^{\hat{I} \hat{J} \hat{K} \hat{L}} H^{\hat{K} \hat{L} \hat{M} \hat{N}}=d_{\mathcal{N}=6} \frac{1}{X}\left(\varepsilon^{\hat{I} \hat{J} \hat{M} \hat{N}} \pm \delta^{\hat{I} \hat{M}} \delta^{\hat{J} \hat{N}} \mp \delta^{\hat{I} \hat{N}} \delta^{\hat{J} \hat{M}}\right) \\
&-\frac{1}{8} d_{\mathcal{N}=6} \frac{A^{\hat{P} \hat{Q}} A^{\hat{R} \hat{S}}}{X^{5}} \varepsilon^{\hat{P} \hat{Q} \hat{R} \hat{S}}\left(\delta^{\hat{I} \hat{M}} \delta^{\hat{J} \hat{N}}-\delta^{\hat{J} \hat{M}} \delta^{\hat{I} \hat{N}} \pm \varepsilon^{\hat{I} \hat{J} \hat{M} \hat{N}}\right) \\
&+\frac{1}{2} d_{\mathcal{N}=6} \frac{A^{\hat{P} \hat{Q}}}{X^{3}}\left[\varepsilon^{\hat{J} \hat{M} \hat{P} \hat{Q}} \delta^{\hat{I} \hat{N}}+\varepsilon^{\hat{I} \hat{N} \hat{P} \hat{Q}} \delta^{\hat{J} \hat{M}}-\varepsilon^{\hat{J} \hat{N} \hat{P} \hat{Q}} \delta^{\hat{I} \hat{M}}-\varepsilon^{\hat{I} \hat{M} \hat{P} \hat{Q}} \delta^{\hat{J} \hat{N}}\right. \\
&\left. \pm 2 \delta^{\hat{P} \hat{J}}\left(\delta^{\hat{N} \hat{I}} \delta^{\hat{Q} \hat{M}}-\delta^{\hat{M} \hat{I}} \delta^{\hat{Q} \hat{N}}\right) \mp 2 \delta^{\hat{P} \hat{I}}\left(\delta^{\hat{N} \hat{J}} \delta^{\hat{Q} \hat{M}}-\delta^{\hat{M} \hat{J}} \delta^{\hat{Q} \hat{M}}\right)\right]
\end{aligned}
$$

This correlator was found in [2] in another form within the iso-spinor formalism. We point out that in (3.34a) there is no mixed correlator involving both $L_{+}^{\hat{I} \hat{J}}$ and $L_{-}^{\hat{I} \hat{J}}$. 


\section{Discussion}

As continuation of the program initiated in $[1,2]$, in this paper we have computed the twoand three-point correlation functions of the supercurrent multiplets in general $\mathcal{N}=5,6$ superconformal field theories in three dimensions. We demonstrated that the functional form of each of these correlators is completely determined by the superconformal symmetry modulo a single overall coefficient. The ratio of the coefficients arising in the two-point and three-point functions is fixed by the Ward identities. The remaining coefficients are model-dependent.

Every $\mathcal{N}=5$ or $\mathcal{N}=6$ superconformal field theory can be viewed as a special $\mathcal{N}=4$ superconformal field theory. We demonstrated that the general property of the $\mathcal{N}=5$ or $\mathcal{N}=6$ superconformal field theories is that they are invariant under the $\mathcal{N}=4$ mirror map.

As is explained in section 1 , the $\mathcal{N}=5$ supercurrent is described by an iso-vector $J^{I}$ while the $\mathcal{N}=6$ supercurrent is given by an antisymmetric tensor $J^{I J}$. As a consequence, their three-point correlation functions are specified by rank-3 $H^{I J K}$ and rank-6 $H^{I J K L M N}$ tensors, respectively. Although the form of the tensor $H^{I J K}$ is relatively compact, see (2.7), the $\mathcal{N}=6$ tensor $H^{I J K L M N}$ has rather clumsy form because of proliferation of $\mathrm{SO}(6)$ indices (3.8). It is desirable to develop a superspace formalism that provides a compact form for these correlators. It is natural to expect that this should be a version of harmonic/projective superspace since supercurrents in such superspaces may be realised as scalar superfields.

A few years ago, ref. [26] presented a family of homogeneous spaces, $\overline{\mathbb{M}}^{3}{ }^{2 \mathcal{N}} \times \mathbb{X}_{m}^{\mathcal{N}}$, of the 3D $\mathcal{N}$-extended superconformal group $\operatorname{OSp}(\mathcal{N} \mid 2, \mathbb{R})$, for any positive integer $m \leq[\mathcal{N} / 2]$, with $[\mathcal{N} / 2]$ the integer part of $\mathcal{N} / 2$. Here $\overline{\mathbb{M}}^{3 \mid 2 \mathcal{N}}$ denotes the compactified $\mathcal{N}$-extended Minkowski superspace on which the superconformal $\operatorname{group} \operatorname{OSp}(\mathcal{N} \mid 2, \mathbb{R})$ acts by well-defined transformations. The usual Minkowski superspace is embedded in $\overline{\mathbb{M}}^{3} \mid 2 \mathcal{N}$ as a dense open subset. The internal sector $\mathbb{X}_{m}^{\mathcal{N}}$ of $\overline{\mathbb{M}}^{3 \mid 2 \mathcal{N}} \times \mathbb{X}_{m}^{\mathcal{N}}$ is realised in terms of odd supertwistors subject to certain conditions [26]. For many applications, it suffices to work with the dense open subset $\mathbb{M}^{3 \mid 2 \mathcal{N}} \times \mathbb{X}_{m}^{\mathcal{N}}$ of $\overline{\mathbb{M}}^{3 \mid 2 \mathcal{N}} \times \mathbb{X}_{m}^{\mathcal{N}}$. Then the points of $\mathbb{X}_{m}^{\mathcal{N}}$ can be identified with $m$ complex $\mathcal{N}$-vectors $Z^{\underline{j}}=\left(Z_{I^{-}}\right) \in \mathbb{C}^{\mathcal{N}}-\{0\}$ which are required to (i) be linearly independent; (ii) obey the null conditions

$$
Z^{\underline{j}} \cdot Z^{\underline{k}}:=Z_{I}^{\underline{j}} Z_{I} \underline{\underline{k}}=0, \quad \forall \underline{j}, \underline{k}=1, \ldots, m ;
$$

and (iii) be defined modulo the equivalence relation

$$
Z_{I^{\underline{j}}} \sim Z_{I^{\underline{k}}} D_{\underline{\underline{k}}}^{\underline{j}}, \quad D=\left(D_{\underline{\underline{k}}}^{\underline{j}}\right) \in \mathrm{GL}(m, \mathbb{C}) .
$$

In the case $\mathcal{N}=3$ and $m=1, \mathbb{M}^{3 \mid 6} \times \mathbb{X}_{1}^{3}$ may be seen to be equivalent to the standard $\mathcal{N}=3$ harmonic superspace $\mathbb{M}^{3 \mid 6} \times \mathbb{C} P^{1}$ [7]. It was shown in [26] that for $\mathcal{N}>2$ and $m=1$ the internal manifold $\mathbb{X}_{1}^{\mathcal{N}}$ is a symmetric space,

$$
\mathbb{X}_{1}^{\mathcal{N}}=\mathrm{SO}(\mathcal{N}) / \mathrm{SO}(\mathcal{N}-2) \times \mathrm{SO}(2), \quad \mathcal{N}>2 .
$$

When dealing with the $\mathcal{N}=5$ supercurrent $J^{I}$, it is natural to make use of the harmonic/projective superspace $\mathbb{M}^{3 \mid 10} \times \mathbb{X}_{1}^{5}$. Using the null five-vector $Z_{I}$ parametrising 
$\mathbb{X}_{1}^{5}$, we introduce the first-order operators

$$
\mathfrak{D}_{\alpha}:=Z_{I} D_{\alpha}^{I}, \quad\left\{\mathfrak{D}_{\alpha}, \mathfrak{D}_{\beta}\right\}=0
$$

and associate with $J^{I}$ the superfield $\mathfrak{J}:=Z_{I} J^{I}$. Then, the supercurrent conservation equation (1.3) implies that $\mathfrak{J}$ is an analytic superfield,

$$
\mathfrak{D}_{\alpha} \mathfrak{J}=0
$$

When dealing with the $\mathcal{N}=6$ supercurrent $J^{I J}=-J^{J I}$, it is natural to make use of the harmonic/projective superspace $\mathbb{M}^{3 \mid 12} \times \mathbb{X}_{2}^{6}$. Using the null six-vectors $Z_{I}$ 를 parametrising $\mathbb{X}_{2}^{6}$, we introduce the first-order operators

$$
\mathfrak{D}^{\frac{j}{\alpha}}:=Z_{I} \underline{\underline{j}} D_{\alpha}^{I}, \quad\left\{\mathfrak{D} \frac{j}{\alpha}, \mathfrak{D} \frac{k}{\beta}\right\}=0, \quad \underline{j}, \underline{k}=\underline{1}, \underline{2}
$$

and associate with $J^{I J}$ the superfield $\mathfrak{J}:=\frac{1}{2} \varepsilon_{\underline{j}} \underline{k} Z_{\bar{I}}^{j} Z_{\bar{J}}^{\frac{k}{J}} J^{I J}$, with $\varepsilon_{\underline{j}} \underline{k}$ an antisymmetric tensor. Then the supercurrent conservation equation (1.5) implies that $\mathfrak{J}$ is an analytic superfield,

$$
\mathfrak{D} \frac{j}{\alpha} \mathfrak{J}=0 .
$$

It is clear that the correlation functions of the $\mathcal{N}=5$ and $\mathcal{N}=6$ supercurrent multiplets should simplify if the above harmonic/projective superspace settings are used. It would be interesting to develop superconformal formalisms to compute correlation functions of primary analytic superfields in such superspaces.

In a recent work [27], a non-standard $\mathcal{N}=6$ harmonic superspace was introduced with the aim to study the three-point correlation functions of BPS operators in $\mathcal{N}=6$ superconformal field theories. It was pointed out that the supercurrent multiplet is a BPS operator, and therefore [27] provided a harmonic-superspace expression for the supercurrent three-point correlator. However, the authors of [27] did not describe how their harmonic superspace is related to the superspaces $\mathbb{M}^{3 \mid 12} \times \mathbb{X}_{m}^{6}$ introduced in [26]. As a result, a precise relationship between the results of [27] and the present paper remains to be understood.

Another possible extension of the present work is the study of four-point correlation functions of conserved currents in three-dimensional superconformal field theories. One can hope that the extended supersymmetry imposes so strong constraints, for sufficiently large $\mathcal{N}$, on the four-point correlators that their form can be found explicitly, as demonstrated for the $4 \mathrm{D} \mathcal{N}=4 \mathrm{SYM}$ theory [28]. We leave these issues for further studies.

\section{Acknowledgments}

We are grateful to Evgeny Buchbinder for useful discussions and to Joseph Novak for comments on the manuscript. This work is supported in part by the ARC DP project DP140103925. 


\section{A Two- and three-point building blocks}

In this appendix we give a brief summary of the two- and three-point superconformal structures $\mathcal{N}$-extended superspace, which were introduced in [1]. These structures have been used in the construction of the supercurrent correlation functions in the main body of the paper.

Consider $\mathcal{N}$-extended Minkowski superspace $\mathbb{M}^{3 \mid 2 \mathcal{N}}$ parametrised by real bosonic $x^{\alpha \beta}=$ $x^{\beta \alpha}$ and fermionic $\theta_{I}^{\alpha}$ coordinates

$$
z^{A}=\left(x^{\alpha \beta}, \theta_{I}^{\alpha}\right), \quad \alpha=1,2, \quad I=1, \ldots, \mathcal{N} .
$$

Here $\alpha, \beta$ are the $\mathrm{SL}(2, \mathbb{R})$ spinor indices, while $I$ is the $R$-symmetry index. All building blocks are composed of the two-point structures

$$
\begin{aligned}
& \boldsymbol{x}_{12}^{\alpha \beta}=\left(x_{1}-x_{2}\right)^{\alpha \beta}+2 \mathrm{i} \theta_{1 I}^{(\alpha} \theta_{2 I}^{\beta)}-\mathrm{i} \theta_{12 I}^{\alpha} \theta_{12 I}^{\beta}, \\
& \theta_{12 I}^{\alpha}=\left(\theta_{1}-\theta_{2}\right)_{I}^{\alpha} .
\end{aligned}
$$

The matrix (A.2a) has the following symmetry property

$$
\boldsymbol{x}_{21}^{\alpha \beta}=-\boldsymbol{x}_{12}^{\beta \alpha} .
$$

A useful object is the square of this matrix

$$
\boldsymbol{x}_{12}^{2}:=-\frac{1}{2} \boldsymbol{x}_{12}^{\alpha \beta} \boldsymbol{x}_{12 \alpha \beta}
$$

One more important two-point structure is the $\mathcal{N} \times \mathcal{N}$ matrix

$$
u_{12}=\left(u_{12}^{I J}\right), \quad u_{12}^{I J}=\delta^{I J}+2 \mathrm{i} \theta_{12}^{\alpha I}\left(\boldsymbol{x}_{12}^{-1}\right)_{\alpha \beta} \theta_{12}^{\beta J},
$$

where

$$
\left(\boldsymbol{x}_{12}^{-1}\right)_{\alpha \beta}=-\frac{\boldsymbol{x}_{12 \beta \alpha}}{\boldsymbol{x}_{12}{ }^{2}}
$$

is the inverse for $\left(\boldsymbol{x}_{12}\right)^{\alpha \beta}$, that is $\left(\boldsymbol{x}_{12}^{-1}\right)_{\alpha \beta}\left(\boldsymbol{x}_{12}\right)^{\beta \gamma}=\delta_{\alpha}^{\gamma}$. One may check that the matrix $u_{12}$ is orthogonal and unimodular,

$$
u_{12}^{\mathrm{T}} u_{12}=\mathbb{1}_{\mathcal{N}}, \quad \operatorname{det} u_{12}=1 .
$$

As is shown in [1], the two-point structures (A.2a), (A.4) and (A.5) transform covariantly under the superconformal group i.e., as the tensors with Lorentz and $\mathrm{SO}(\mathcal{N})$ indices at both superspace points. Here we do not give their transformation laws referring the readers to our previous works $[1,2]$.

Associated with three superspace points $z_{1}, z_{2}$ and $z_{3}$ are the following three-point structures:

$$
\begin{aligned}
\boldsymbol{X}_{1 \alpha \beta} & =-\left(\boldsymbol{x}_{21}^{-1}\right)_{\alpha \gamma} \boldsymbol{x}_{23}^{\gamma \delta}\left(\boldsymbol{x}_{13}^{-1}\right)_{\delta \beta}, \\
\Theta_{1 \alpha}^{I} & =\left(\boldsymbol{x}_{21}^{-1}\right)_{\alpha \beta} \theta_{12}^{I \beta}-\left(\boldsymbol{x}_{31}^{-1}\right)_{\alpha \beta} \theta_{13}^{I \beta}, \\
U_{1}^{I J} & =u_{12}^{I K} u_{23}^{K L} u_{31}^{L J} .
\end{aligned}
$$


These objects are labeled by the index 1 reflecting the fact that they transform as tensors in the superspace point $z_{1}$. Performing the cyclic permutation of superspace points $z_{1}, z_{2}$ and $z_{3}$ one can obtain similar objects transforming as tensors in points $z_{2}$ and $z_{3}$. The three-point structures at different superspace points are related to each other as follows

$$
\begin{aligned}
\boldsymbol{x}_{13}^{\alpha \alpha^{\prime}} \boldsymbol{X}_{3 \alpha^{\prime} \beta^{\prime}} \boldsymbol{x}_{31}^{\beta^{\prime} \beta} & =-\left(\boldsymbol{X}_{1}^{-1}\right)^{\beta \alpha}=\frac{\boldsymbol{X}_{1}^{\alpha \beta}}{\boldsymbol{X}_{1}^{2}}, \\
\Theta_{1 \gamma}^{I} \boldsymbol{x}_{13}^{\gamma \delta} \boldsymbol{X}_{3 \delta \beta} & =u_{13}^{I J} \Theta_{3 \beta}^{J}, \\
U_{3}^{I J} & =u_{31}^{I K} U_{1}^{K L} u_{13}^{L J} .
\end{aligned}
$$

The three-point structures (A.8) have several important properties. In particular, the tensor (A.8a) can be decomposed into symmetric and antisymmetric parts

$$
\boldsymbol{X}_{\alpha \beta}=X_{\alpha \beta}-\frac{\mathrm{i}}{2} \varepsilon_{\alpha \beta} \Theta^{2}
$$

where the symmetric spinor $X_{\alpha \beta}=X_{\beta \alpha}$ is equivalently represented as a three-vector $X_{m}=$ $-\frac{1}{2} \gamma_{m}^{\alpha \beta} X_{\alpha \beta}$. Here and below we suppress the subscript labelling the superspace point.

Next, the matrix (A.8c) can be expressed in terms of (A.8a) and (A.8b) similarly to (A.5):

$$
U^{I J}=\delta^{I J}+2 \mathrm{i} \Theta_{\alpha}^{I}\left(\boldsymbol{X}^{-1}\right)^{\alpha \beta} \Theta_{\beta}^{J}=\delta^{I J}-2 \frac{A^{I J}}{\boldsymbol{X}^{2}}+\frac{\Theta^{I \alpha} \Theta_{\alpha}^{J} \Theta^{2}}{\boldsymbol{X}^{2}}
$$

where

$$
A^{I J}=\mathrm{i} \Theta^{I \alpha} X_{\alpha \beta} \Theta^{J \beta}
$$

We point out that the three-point objects (A.8) look like local expressions. In fact, in computing correlators we consider functions of these objects obeying certain differential equations. These differential equations involve generalised superspace derivatives such as

$$
\mathcal{D}_{\alpha}^{I}=\frac{\partial}{\partial \Theta_{I}^{\alpha}}+\mathrm{i} \gamma_{\alpha \beta}^{m} \Theta^{I \beta} \frac{\partial}{\partial X^{m}}
$$

This derivative should not be confused with the usual superspace derivative $D_{\alpha}^{I}=\frac{\partial}{\partial \theta_{I}^{\alpha}}+$ $\mathrm{i} \gamma_{\alpha \beta}^{m} \theta^{I \beta} \frac{\partial}{\partial x^{m}}$ which acts on the superspace coordinates (A.1). The anticommutation relations for these derivatives are

$$
\left\{D_{\alpha}^{I}, D_{\beta}^{J}\right\}=2 \mathrm{i} \delta^{I J} \partial_{\alpha \beta},
$$

and similar for the generalised ones (A.13).

Open Access. This article is distributed under the terms of the Creative Commons Attribution License (CC-BY 4.0), which permits any use, distribution and reproduction in any medium, provided the original author(s) and source are credited. 


\section{References}

[1] E.I. Buchbinder, S.M. Kuzenko and I.B. Samsonov, Superconformal field theory in three dimensions: Correlation functions of conserved currents, JHEP 06 (2015) 138 [arXiv: 1503.04961] [INSPIRE].

[2] E.I. Buchbinder, S.M. Kuzenko and I.B. Samsonov, Implications of $\mathcal{N}=4$ superconformal symmetry in three spacetime dimensions, JHEP 08 (2015) 125 [arXiv:1507.00221] [INSPIRE].

[3] K. Hosomichi, K.-M. Lee, S. Lee, S. Lee and J. Park, $N=$ 5, 6 Superconformal Chern-Simons Theories and M2-branes on Orbifolds, JHEP 09 (2008) 002 [arXiv:0806.4977] [INSPIRE].

[4] O. Aharony, O. Bergman, D.L. Jafferis and J. Maldacena, $N=6$ superconformal Chern-Simons-matter theories, M2-branes and their gravity duals, JHEP 10 (2008) 091 [arXiv:0806.1218] [INSPIRE].

[5] O. Aharony, O. Bergman and D.L. Jafferis, Fractional M2-branes, JHEP 11 (2008) 043 [arXiv:0807.4924] [INSPIRE].

[6] I.L. Buchbinder, E.A. Ivanov, O. Lechtenfeld, N.G. Pletnev, I.B. Samsonov and B.M. Zupnik, ABJM models in $N=3$ harmonic superspace, JHEP 03 (2009) 096 [arXiv:0811.4774] [INSPIRE].

[7] B.M. Zupnik and D.V. Khetselius, Three-dimensional extended supersymmetry in the harmonic superspace (in Russian), Sov. J. Nucl. Phys. 47 (1988) 730 [INSPIRE].

[8] J. Bagger and N. Lambert, Modeling Multiple M2's, Phys. Rev. D 75 (2007) 045020 [hep-th/0611108] [INSPIRE].

[9] J. Bagger and N. Lambert, Gauge symmetry and supersymmetry of multiple M2-branes, Phys. Rev. D 77 (2008) 065008 [arXiv:0711.0955] [INSPIRE].

[10] A. Gustavsson, Algebraic structures on parallel M2-branes, Nucl. Phys. B 811 (2009) 66 [arXiv:0709.1260] [INSPIRE].

[11] S. Ferrara and B. Zumino, Transformation Properties of the Supercurrent, Nucl. Phys. B 87 (1975) 207 [InSPIRE].

[12] D. Butter, S.M. Kuzenko, J. Novak and G. Tartaglino-Mazzucchelli, Conformal supergravity in three dimensions: New off-shell formulation, JHEP 09 (2013) 072 [arXiv:1305.3132] [INSPIRE].

[13] S.M. Kuzenko, J. Novak and G. Tartaglino-Mazzucchelli, $N=6$ superconformal gravity in three dimensions from superspace, JHEP 01 (2014) 121 [arXiv:1308.5552] [INSPIRE].

[14] Y. Korovin, S.M. Kuzenko and S. Theisen, The conformal supercurrents in diverse dimensions and conserved superconformal currents, JHEP 05 (2016) 134 [arXiv: 1604.00488] [INSPIRE].

[15] W. Siegel, Unextended Superfields in Extended Supersymmetry, Nucl. Phys. B 156 (1979) 135 [InSPIRE].

[16] N.J. Hitchin, A. Karlhede, U. Lindström and M. Roček, HyperKähler Metrics and Supersymmetry, Commun. Math. Phys. 108 (1987) 535 [INSPIRE].

[17] B.M. Zupnik and D.G. Pak, Superfield Formulation of the Simplest Three-dimensional Gauge Theories and Conformal Supergravities, Theor. Math. Phys. 77 (1988) 1070 [InSPIRE]. 
[18] H. Samtleben and R. Wimmer, $N=6$ Superspace Constraints, SUSY Enhancement and Monopole Operators, JHEP 10 (2010) 080 [arXiv:1008.2739] [INSPIRE].

[19] S.M. Kuzenko, U. Lindström and G. Tartaglino-Mazzucchelli, Off-shell supergravity-matter couplings in three dimensions, JHEP 03 (2011) 120 [arXiv:1101.4013] [INSPIRE].

[20] U. Gran, J. Greitz, P.S. Howe and B.E.W. Nilsson, Topologically gauged superconformal Chern-Simons matter theories, JHEP 12 (2012) 046 [arXiv:1204.2521] [INSPIRE].

[21] S.M. Kuzenko and J. Novak, Supergravity-matter actions in three dimensions and Chern-Simons terms, JHEP 05 (2014) 093 [arXiv: 1401.2307] [INSPIRE].

[22] H. Osborn, $N=1$ superconformal symmetry in four-dimensional quantum field theory, Annals Phys. 272 (1999) 243 [hep-th/9808041] [INSPIRE].

[23] S.M. Kuzenko and S. Theisen, Correlation functions of conserved currents in $N=2$ superconformal theory, Class. Quant. Grav. 17 (2000) 665 [hep-th/9907107] [INSPIRE].

[24] J.-H. Park, Superconformal symmetry in three-dimensions, J. Math. Phys. 41 (2000) 7129 [hep-th/9910199] [INSPIRE].

[25] S.M. Kuzenko and I.B. Samsonov, Superconformal Chern-Simons-matter theories in $\mathcal{N}=4$ superspace, Phys. Rev. D 92 (2015) 105007 [arXiv:1507.05377] [INSPIRE].

[26] S.M. Kuzenko, J.-H. Park, G. Tartaglino-Mazzucchelli and R. Unge, Off-shell superconformal nonlinear $\sigma$-models in three dimensions, JHEP 01 (2011) 146 [arXiv:1011.5727] [INSPIRE].

[27] P. Liendo, C. Meneghelli and V. Mitev, On correlation functions of BPS operators in $3 d$ $\mathcal{N}=6$ superconformal theories, arXiv:1512.06072 [INSPIRE].

[28] G.P. Korchemsky and E. Sokatchev, Four-point correlation function of stress-energy tensors in $\mathcal{N}=4$ superconformal theories, JHEP 12 (2015) 133 [arXiv:1504.07904] [INSPIRE]. 